\title{
Fierz-complete NJL model study. III. Emergence from quark-gluon dynamics
}

\author{
Jens Braun, ${ }^{1,2}$ Marc Leonhardt, ${ }^{1}$ and Martin Pospiech ${ }^{1}$ \\ ${ }^{1}$ Institut für Kernphysik (Theoriezentrum), Technische Universität Darmstadt, \\ D-64289 Darmstadt, Germany \\ ${ }^{2}$ ExtreMe Matter Institute EMMI, GSI, Planckstraße 1, D-64291 Darmstadt, Germany
}

(Received 27 September 2019; accepted 2 January 2020; published 10 February 2020)

\begin{abstract}
Our understanding of the dynamics and the phase structure of dense strong-interaction matter is to a large extent still built on the analysis of low-energy models, such as those of the Nambu-Jona-Lasinio-type model. In this work, we analyze the emergence of the latter class of models at intermediate- and low-energy scales from fundamental quark-gluon interactions. To this end, we study the renormalization group flow of a Fierz-complete set of four-quark interactions and monitor their strength at finite temperature and quark chemical potential. At small quark chemical potential, we find that the scalar-pseudoscalar interaction channel is dynamically rendered most dominant by the gauge degrees of freedom, indicating the formation of a chiral condensate. Moreover, the inclusion of quark-gluon interactions leaves a significant imprint on the dynamics as measured by the curvature of the finite-temperature phase boundary which we find to be in accordance with lattice QCD results. At large quark chemical potential, we then observe that the dominance pattern of the four-quark couplings is changed by the underlying quark-gluon dynamics, without any finetuning of the four-quark couplings. In this regime, the scalar-pseudoscalar interaction channel becomes subleading, and the dominance pattern suggests the formation of a chirally symmetric diquark condensate. In particular, our study confirms the importance of explicit $U_{\mathrm{A}}(1)$ breaking for the formation of this type of condensate at high densities.
\end{abstract}

DOI: 10.1103/PhysRevD.101.036004

\section{INTRODUCTION}

Low-energy models of the theory of the strong interaction (quantum chromodynamics, QCD) are still considered very valuable for a variety of reasons. In particular, in the high-density regime, which is at least difficult to access with lattice Monte Carlo techniques, the Nambu-JonaLasinio (NJL) model $[1,2]$ and its various variations and relatives (see, e.g., Refs. [3-8] for reviews), such as quarkmeson (QM) models, allow us to gain some insight into the plethora of symmetry-breaking patterns that may potentially be realized in this regime; see Refs. [9-12] for reviews. From a phenomenological point of view, this regime attracts significant interest from the astrophysics side. For example, studies of neutron stars require a knowledge of the equation of state of strong-interaction matter as input. However, the latter are currently still plagued by (significant) uncertainties, at least at high density; see, e.g., Ref. [13] for a recent review. In order

Published by the American Physical Society under the terms of the Creative Commons Attribution 4.0 International license. Further distribution of this work must maintain attribution to the author(s) and the published article's title, journal citation, and DOI. Funded by SCOAP . to constrain the equation of state further [14], we eventually need a better understanding of the symmetry-breaking patterns of QCD guided by first-principle approaches.

In two preceding works $[15,16]$, we have studied the relevance of Fierz completeness of four-quark selfinteractions in NJL-type models at finite temperature and quark chemical potential. Our beyond-mean-field renormalization-group (RG) analysis of the "hierarchy" of the various interaction channels in terms of their relative strengths allowed us to gain insight into the symmetrybreaking patterns and the structure of the ground state. Particularly at high density, we found the aspect of Fierz completeness to be of great importance, leading to an increased phase transition temperature as compared to conventional NJL model studies. At least naively, this observation might have crucial implications for the properties of cold dense quark matter at low temperatures as an increase of the critical temperature may point to an increase of the size of the gap in the low-energy spectrum.

The four-quark couplings appearing in the ansatz of NJL-type models are usually considered as fundamental parameters. In fact, owing to the nonrenormalizability of NJL-type models in four space-time dimensions, both on the perturbative and on the nonperturbative level (see, e.g., Refs. $[17,18]$ ), the ultraviolet (UV) cutoff scale $\Lambda$ becomes 
a parameter of the model, too. Against this background, the regularization scheme also becomes part of the definition of the model. The initial values of the four-quark couplings are then chosen such that a given set of lowenergy observables is reproduced in the vacuum limit. In Ref. [16], guided by the findings of RG studies of QCD [19-21] and in order to relate to conventional NJL model studies, we have set all four-quark couplings to zero initially, except for the scalar-pseudoscalar coupling. As the only remaining parameter, we adjusted this coupling to fix low-energy observables in the vacuum limit. However, this scale-fixing procedure underlying many low-energy model studies can be potentially problematic. The distinct role of the scalar-pseudoscalar interaction channel at the initial UV cutoff scale can indeed be questioned since a specific four-quark interaction channel is reducible by means of Fierz transformations. Yet adopting more complex initial conditions by also taking into account four-quark couplings other than the scalar-pseudoscalar interaction channel may suffer from the fact that the parameters cannot be uniquely determined by a given set of low-energy observables. Indeed, the values of the low-energy observables may, in general, be reproduced by various different parameter sets, or certain parameters might even be left undetermined. The remaining ambiguity has been found to affect the phase structure significantly; see, e.g., Refs. [9,22-24]. Moreover, boundary conditions which are defined in the vacuum limit are possibly inappropriate for computations at finite external control parameters, such as temperature $T$ and/or quark chemical potential $\mu$. Considering NJL-type models to be rooted in $\mathrm{QCD}$, the RG evolution of gluon-induced four-quark interactions in fact suggests a dependence of these model parameters on external control parameters [25]. In particular, at finite quark chemical potential, as also discussed in detail in Refs. [15,16] (see, e.g., Refs. [9,11,12,26] for reviews), effective degrees of freedom (d.o.f.) associated with four-quark interaction channels other than the scalarpseudoscalar interaction channel are expected to become important or even dominant. In such a situation, a choice for the initial conditions with a specifically tuned scalarpseudoscalar coupling is therefore unfortunate as it may potentially bias the outcome in terms of symmetry-breaking patterns along the finite-temperature phase boundary.

Thus far, we have not yet discussed the role of the UV cutoff scale. In the context of NJL-type models, we have to deal with the existence of a finite UV extent; i.e., the cutoff scale $\Lambda$ is limited by a validity bound which in turn limits the model's range of applicability in terms of external parameters. The origin of this bound is actually twofold: First, NJL-type models eventually become unstable in the UV and develop a Landau pole at a certain scale. Second, these models have a phenomenological UV extent beyond which the description of the physics in terms of the models' effective fields becomes invalid and the knowledge of the underlying fundamental dynamics, i.e., quark-gluon dynamics, is ultimately required. As a consequence, a choice of the UV cutoff scale within the a priori unknown validity bound either limits the applicable range of external parameters or, for external parameters outside of this range, implies that the initial effective action is already a complicated object itself; see Ref. [27] for a detailed discussion of these issues. Considering NJL-type models to be embedded in QCD, a possibility to resolve this problem is the determination of the model parameters by employing RG studies of the fundamental theory; see, e.g., Refs. [25,28,29]. In the past 20 years, tremendous progress has been made within the functional RG framework in the development of a "topdown" approach to QCD; see, e.g., Refs. [20,21,30-50]. The only input in such an approach is given by the fundamental parameters of QCD, i.e., the current quark masses and the value of the strong coupling in the perturbative high-momentum regime. These functional RG studies are basically free of additional model parameters. In this context, even very good quantitative agreement of results from lattice QCD and functional RG studies has been demonstrated at zero and finite temperature for QCD with different flavor numbers; see, e.g., Refs. [21,35,37,38,50].

Whereas the aforementioned RG studies aiming at quantitative precision constitute essential advances towards predictive first-principle investigations of the QCD phase diagram, eventually, even at high densities, we aim to take another important step toward such a top-down firstprinciples approach to analyze the phase structure of QCD at high densities with functional methods. With our analysis of the Fierz-complete NJL model with two quark flavors in Ref. [16], we have gained valuable insight into the quark dynamics. In the present work, we now incorporate gluodynamics by extending our Fierz-complete ansatz with dynamical gauge d.o.f., following earlier functional RG studies $[36,37,39,45]$. In full QCD, the values of the four-quark couplings are no longer fundamental parameters since these self-interactions are fluctuation induced by the dynamics of the gauge fields. Taking this aspect into account, the aforementioned issue associated with the determination of model parameters - such as ambiguities related to the possibility to Fierz transform given initial conditions and the potential existence of more than one parameter set reproducing equally well a given set of low-energy observables, or the dependence of the initial conditions on external control parameters-can, in principle, be resolved. More specifically, including gauge dynamics and thus resolving the fundamental microscopic d.o.f. allows the initialization of the $\mathrm{RG}$ flow at a large scale $\Lambda$ associated with the perturbative regime, which effectively corresponds to starting in the vacuum as we have $T / \Lambda \ll 1$ and $\mu / \Lambda \ll 1$. In this way, the finite UV extent as implied by the validity bound of NJL-type models is surmounted, and the limit on the range of applicability in terms of external parameters is lifted. 
Working in the chiral limit, the strong coupling $g_{\mathrm{s}}$ is the only parameter which is set at the initial UV scale $\Lambda$. By integrating out fluctuations from this scale $\Lambda$, the quarkgluon vertex gives rise to 1PI box diagrams with two-gluon exchange, which dynamically generate the four-quark interaction channels. Depending on the strength of the strong coupling and the external parameters, the quark sector may then be driven to criticality, signaling the onset of spontaneous symmetry breaking, e.g., chiral symmetry breaking or diquark condensation of a specific type. Following the approach developed in our two preceding works [15,16], we consider the RG flow of the four-quark couplings in the pointlike limit to study the QCD phase structure at finite temperature and quark chemical potential. In particular, we analyze the "hierarchy" of the four-quark couplings in terms of their strength, which shall prove very valuable in order to gain insight into the symmetry properties of the QCD ground state in the low-energy limit.

This work is organized as follows: In Sec. II, we discuss general aspects of the formalism and concepts underlying our study. We begin with a discussion of the relation of the quark-gluon vertex and four-quark interactions in Sec. II A. In Sec. II B, we then briefly review the relation of the RG flow of four-fermion couplings to the onset of phase transitions, including a discussion of the general structure of the RG flow equations for the four-quark couplings. The scale-fixing procedure underlying our present work is discussed in Sec. IIC. The QCD phase structure and symmetry-breaking patterns at finite temperature and density are then analyzed in Sec. III. There, we also compare the results for the phase boundary to the one obtained from our previous Fierz-complete NJL model study [16]. Moreover, we discuss the effect of explicit $U_{\mathrm{A}}(1)$ symmetry breaking and comment on the curvature of the finite-temperature phase boundary at small chemical potential resulting from various different approaches. Our conclusions can be found in Sec. IV.

\section{GENERAL ASPECTS OF THE FORMALISM}

\section{A. Quark-gluon vertex and four-quark interactions}

In the present work, we employ the functional RG approach [51] to study the RG flow of QCD starting from the Euclidean QCD action (see Refs. [17,52,53] for reviews):

$$
S=\int \mathrm{d}^{4} x\left\{\frac{1}{4} F_{\mu \nu}^{a} F_{\mu \nu}^{a}+\bar{\psi}\left(\mathrm{i} \not \partial+\bar{g}_{\mathrm{s}} A+\mathrm{i} \gamma_{0} \mu\right) \psi\right\},
$$

where $\bar{g}_{\mathrm{s}}$ is the bare gauge coupling and $\mu$ is the quark chemical potential. The gluon fields $A_{\mu}^{a}$ enter the definition of the field-strength tensor $F_{\mu \nu}^{a}$ in the usual way. We emphasize that we exclusively consider the case of quarks coming in $N_{\mathrm{c}}=3$ colors and $N_{\mathrm{f}}=2$ flavors.
In the RG flow, the quark-gluon vertex generates quark self-interactions already at the one-loop level via two-gluon exchange. This gives rise to contributions to the quantum effective action, e.g., of the following form:

$$
\delta \Gamma=\frac{1}{2} \int \mathrm{d}^{4} x \sum_{j \in \mathcal{B}} Z_{j} \bar{\lambda}_{j} \mathcal{L}_{j}
$$

where the elements $\mathcal{L}_{j}$ form a ten-component Fierzcomplete basis $\mathcal{B}$ of pointlike four-quark interactions to be specified below. The various terms are associated with corresponding bare couplings $\bar{\lambda}_{i}$ and vertex renormalization factors $Z_{j}$. By construction, the couplings are not parameters of our calculation but solely generated by quark-gluon dynamics. This is an important difference from, e.g., NJLtype model studies where such couplings represent input parameters.

In the following, we focus on the RG flow of pointlike projected four-quark correlation functions $\Gamma^{(4)}$, which eventually corresponds to a calculation of the effective action at leading order of the derivative expansion. To be specific, we define the four-quark couplings associated with the vertex of the form (2) as follows:

$$
\begin{aligned}
Z_{j} \bar{\lambda}_{j} \mathcal{L}_{j}= & \lim _{p_{i} \rightarrow 0} \bar{\psi}_{\alpha}\left(p_{1}\right) \bar{\psi}_{\beta}\left(p_{2}\right) \Gamma_{\mathcal{L}_{j}}^{(4), \alpha \beta \gamma \delta}\left(p_{1}, p_{2}, p_{3}, p_{4}\right) \\
& \times \psi_{\gamma}\left(p_{3}\right) \psi_{\delta}\left(p_{4}\right) .
\end{aligned}
$$

Here, $\alpha, \beta, \gamma, \delta$ denote collective indices for color, flavor, and Dirac structures determined by a specific element $\mathcal{L}_{j}$ of our Fierz-complete basis. We add that this zero-momentum projection does not represent a Silver Blaze symmetric point $[15,54,55]$. However, it matches the standard definition of four-quark couplings in conventional low-energy models (see Refs. [3,4,9,10,56] for reviews) and BCS-type models (see Refs. [11,12,26,57] for reviews).

Let us now specify the elements $\mathcal{L}_{j}$ of our Fierzcomplete basis $\mathcal{B}$ of pointlike four-quark interactions. Since Poincaré invariance is explicitly broken in our calculations at finite temperature and quark chemical potential, we are only left with rotational invariance among the spatial components of the various possible channels. Moreover, a finite quark chemical potential also explicitly breaks the charge conjugation symmetry. Therefore, with respect to the fundamental symmetries associated with charge conjugation, time reversal, and parity, only invariance under parity transformations and time-reversal transformations remains intact. Assuming invariance of the channels under $S U\left(N_{\mathrm{c}}\right) \otimes S U_{\mathrm{L}}(2) \otimes S U_{\mathrm{R}}(2) \otimes U_{\mathrm{V}}(1)$, we end up with a Fierz-complete basis composed of ten elements [16]. Guided by QCD low-energy phenomenology, we choose four of the ten channels such that they are invariant under $S U\left(N_{\mathrm{c}}\right) \otimes S U_{\mathrm{L}}(2) \otimes S U_{\mathrm{R}}(2) \otimes U_{\mathrm{V}}(1)$ transformations but break the $U_{\mathrm{A}}(1)$ symmetry explicitly: 


$$
\begin{gathered}
\mathcal{L}_{(\sigma-\pi)}=(\bar{\psi} \psi)^{2}-\left(\bar{\psi} \gamma_{5} \tau_{i} \psi\right)^{2}, \\
\mathcal{L}_{(S+P)_{-}=}(\bar{\psi} \psi)^{2}-\left(\bar{\psi} \gamma_{5} \tau_{i} \psi\right)^{2}+\left(\bar{\psi} \gamma_{5} \psi\right)^{2}-\left(\bar{\psi} \tau_{i} \psi\right)^{2}, \\
\mathcal{L}_{\mathrm{csc}}=4\left(\mathrm{i} \bar{\psi} \gamma_{5} \tau_{2} T^{A} \psi{ }^{C}\right)\left(\mathrm{i} \bar{\psi}{ }^{C} \gamma_{5} \tau_{2} T^{A} \psi\right), \\
\mathcal{L}_{(S+P)_{-}^{\mathrm{adj}}}=\left(\bar{\psi} T^{a} \psi\right)^{2}-\left(\bar{\psi} \gamma_{5} \tau_{i} T^{a} \psi\right)^{2} \\
+\left(\bar{\psi} \gamma_{5} T^{a} \psi\right)^{2}-\left(\bar{\psi} \tau_{i} T^{a} \psi\right)^{2},
\end{gathered}
$$

where, e.g., $\left(\bar{\psi} \gamma_{5} \tau_{i} \psi\right)^{2} \equiv\left(\bar{\psi} \gamma_{5} \tau_{i} \psi\right)\left(\bar{\psi} \gamma_{5} \tau_{i} \psi\right)$ and the $T^{a}$ 's denote the generators of $S U\left(N_{\mathrm{c}}\right)$. Moreover, we introduced charge conjugated fields $\psi^{C}=\mathcal{C} \bar{\psi}^{T}$ and $\bar{\psi}^{C}=\psi^{T} \mathcal{C}$, with $\mathcal{C}=\mathrm{i} \gamma_{2} \gamma_{0}$ being related to the charge conjugation operator. The remaining six channels can then be chosen to be invariant under $S U\left(N_{\mathrm{c}}\right) \otimes S U_{\mathrm{L}}(2) \otimes S U_{\mathrm{R}}(2) \otimes U_{\mathrm{V}}(1) \otimes$ $U_{\mathrm{A}}(1)$ transformations:

$$
\begin{gathered}
\mathcal{L}_{(V+A)_{\|}}=\left(\bar{\psi} \gamma_{0} \psi\right)^{2}+\left(\bar{\psi} \mathrm{i} \gamma_{0} \gamma_{5} \psi\right)^{2}, \\
\mathcal{L}_{(V+A)_{\perp}}=\left(\bar{\psi} \gamma_{i} \psi\right)^{2}+\left(\bar{\psi} \mathrm{i} \gamma_{i} \gamma_{5} \psi\right)^{2}, \\
\mathcal{L}_{(V-A)_{\|}}=\left(\bar{\psi} \gamma \gamma_{0} \psi\right)^{2}-\left(\bar{\psi} \mathrm{i} \gamma_{0} \gamma_{5} \psi\right)^{2}, \\
\mathcal{L}_{(V-A)_{\perp}}=\left(\bar{\psi} \gamma_{i} \psi\right)^{2}-\left(\bar{\psi} \mathrm{i} \gamma_{i} \gamma_{5} \psi\right)^{2}, \\
\mathcal{L}_{(V+A)_{\|}^{\mathrm{adj}}}=\left(\bar{\psi} \gamma_{0} T^{a} \psi\right)^{2}+\left(\bar{\psi} \mathrm{i} \gamma_{0} \gamma_{5} T^{a} \psi\right)^{2}, \\
\mathcal{L}_{(V-A)_{\perp} \text { adj }}=\left(\bar{\psi} \gamma_{i} T^{a} \psi\right)^{2}-\left(\bar{\psi} \mathrm{i} \gamma_{i} \gamma_{5} T^{a} \psi\right)^{2} .
\end{gathered}
$$

Of course, this basis is not unique. In principle, we can combine elements of the basis to perform a basis transformation. However, as indicated above, our present choice is motivated by the structure of the four-quark channels conventionally employed in QCD low-energy models. In fact, the scalar-pseudoscalar channel associated with pion dynamics and chiral symmetry breaking is given by the channel $\mathcal{L}_{(\sigma-\pi)}$. The channel associated with the element $\mathcal{L}_{(S+P)_{-}}$can be rewritten as (up to a numerical constant)

$$
\sim \operatorname{det}\left(\bar{\psi}\left(1+\gamma_{5}\right) \psi\right)+\operatorname{det}\left(\bar{\psi}\left(1-\gamma_{5}\right) \psi\right),
$$

where the determinant is taken in flavor space. This channel is associated with the presence of topologically nontrivial gauge configurations violating the $U_{\mathrm{A}}(1)$ symmetry. Indeed, such configurations can be recast into a four-quark interaction channel of the form (14) in the case of twoflavor QCD [58-64]. The channel (7) may be viewed as a version of the channel $\mathcal{L}_{(S+P)_{-}}$with a nontrivial color structure. We add that, for the phenomenologically more relevant three-flavor case, the explicit breaking of the $U_{\mathrm{A}}(1)$ symmetry was suggested before the two-flavor case in order to explain the mass splitting of the $\eta$ and $\eta^{\prime}$ in the mesonic mass spectrum $[65,66]$.
Finally, we have included a channel which allows us to "measure" the status of the formation of a diquark condensate of the type

$$
\delta^{a} \sim\left\langle\mathrm{i} \bar{\psi}^{C} \gamma_{5} \epsilon_{f} \varepsilon_{c}^{a} \psi\right\rangle,
$$

which carries a net baryon and net color charge. ${ }^{1}$ Note that the channel $\mathcal{L}_{\text {csc }}$ is invariant under $S U\left(N_{\mathrm{c}}\right) \otimes S U_{\mathrm{L}}(2) \otimes$ $S U_{\mathrm{R}}(2) \otimes U_{\mathrm{V}}(1)$ transformations, and the corresponding condensate leaves the chiral symmetry intact. However, the formation of such a diquark condensate comes along with the breakdown of the $U_{\mathrm{V}}(1)$ symmetry, as expected for a BCS-type condensate. ${ }^{2}$

With respect to our discussion of the effect of $U_{\mathrm{A}}(1)$ symmetry breaking, we add that we can use our Fierzcomplete set of pointlike four-quark interactions to monitor the strength of $U_{\mathrm{A}}(1)$ symmetry breaking. Indeed, requiring that the effective action $\Gamma$ is invariant under $U_{\mathrm{A}}(1)$ transformations, we find the following two sum rules, which are satisfied simultaneously if the $U_{\mathrm{A}}(1)$ symmetry is intact:

$$
\begin{gathered}
\mathcal{S}_{U_{\mathrm{A}}(1)}^{(1)}=\bar{\lambda}_{\mathrm{csc}}+\bar{\lambda}_{(S+P)_{-}^{\mathrm{adj}}}=0, \\
\mathcal{S}_{U_{\mathrm{A}}(1)}^{(2)}=\bar{\lambda}_{(S+P)_{-}}-\frac{N_{\mathrm{c}}-1}{2 N_{\mathrm{c}}} \bar{\lambda}_{\mathrm{csc}}+\frac{1}{2} \bar{\lambda}_{(\sigma-\pi)}=0 ;
\end{gathered}
$$

see Ref. [16] for a more detailed discussion. We shall come back to the issue of $U_{\mathrm{A}}(1)$ symmetry breaking in Sec. III B.

To summarize, from here on, we consider the RG flow of pointlike four-quark interactions as generated by the quarkgluon vertex. The latter implies that the initial conditions of the four-quark interactions are set to zero at the initial RG scale $\Lambda$; i.e., they do not represent parameters of our study as is the case for QCD low-energy models. We emphasize again that our set of four-quark interactions is Fierz complete; i.e., any other pointlike four-quark interaction invariant under the symmetries specified above is reducible by means of Fierz transformations.

Since we consider the RG flow of the four-quark interactions at leading order of the derivative expansion (i.e., in the pointlike limit as indicated above), the quark wave-function renormalization factors do not receive contributions directly from the four-quark interactions; see Ref. [17] for a detailed discussion. Contributions to the quark wave-function renormalizations resulting from the coupling of the quarks to the gluons have been found to be small at this order in earlier studies [32,35,39], at least for

\footnotetext{
${ }^{1}$ Here, $\epsilon_{f} \equiv \epsilon_{f}^{(\alpha, \beta)}$ and $\varepsilon_{c}^{a} \equiv \varepsilon_{c}^{a(m, n)}$ are antisymmetric tensors in flavor and color space, respectively.

${ }^{2}$ With respect to the diquark channel, we add that our conventions in Eq. (6) are such that we only sum over the antisymmetric (A) generators of the $S U\left(N_{\mathrm{c}}\right)$ color group. Normalization factors of this channel are chosen according to the standard literature [9].
} 
RG scales relevant for the present work. Therefore, we do not take into account the running of the quark wavefunction renormalization factors; i.e., we set them to 1 and defer their computation to future work. Finally, we note that quark self-interactions of higher order are also generated dynamically in the RG flow. However, at leading order of the derivative expansion, they do not contribute to the RG flow of the four-quark self-interactions and are thus not included in our present study [15-17]. Based on these general considerations of the symmetries of QCD and the structure of RG flow equations in this subsection, we employ the following Fierz-complete ansatz of the effective action for our computation of the four-quark couplings at leading order $(\mathrm{LO})$ of the derivative expansion:

$$
\begin{aligned}
\Gamma_{\mathrm{LO}}\left[A_{\mu}^{a}, \bar{\psi}, \psi\right]= & \int \mathrm{d}^{4} x\left\{\frac{1}{4} F_{\mu \nu}^{a} F_{\mu \nu}^{a}+\bar{\psi}\left(\mathrm{i} \not \partial+\bar{g}_{\mathrm{s}} \not A+\mathrm{i} \gamma_{0} \mu\right) \psi\right. \\
& \left.+\frac{1}{2} \sum_{j \in \mathcal{B}} Z_{j} \bar{\lambda}_{j} \mathcal{L}_{j}\right\}
\end{aligned}
$$

The initial condition for the RG flow of this ansatz for the effective action is given by Eq. (1), which is nothing but our ansatz for the effective action with all four-quark couplings set to zero. Note that the RG flow equations of the fourquark couplings derived from this ansatz depend on the strong coupling. Thus, we also need to take into account the running of the latter. We shall discuss this in the next subsection.

\section{B. Phase transitions and four-quark interactions}

The four-quark couplings depend on the chemical potential, the temperature, and the RG scale $k$. Although the scale dependence implies that part of the information on the momentum dependence is still taken into account in our RG analysis in an effective manner [67], the pointlike approximation underlying our present work ignores relevant information of four-quark correlation functions. To be more specific, bound-state information is encoded in the momentum structure of the quark correlation functions. Therefore, our present approximation only allows us to study the symmetric high-energy regime [17], whereas the low-energy regime is only accessible in the absence of (spontaneous) symmetry breaking, which is associated with bound-state formation, as is the case at, e.g., high temperature. For our purposes, this is nevertheless sufficient as it enables us to study the approach towards the symmetrybroken low-energy regime. Indeed, symmetry breaking is ultimately triggered by a specific four-quark channel approaching criticality as indicated by a divergence of the corresponding coupling. Such a seeming Landau-poletype behavior of four-quark couplings can be traced back to the formation of condensates since the pointlike fourquark couplings can be shown to be proportional to the inverse mass parameters of a generalized Ginzburg-Landau effective potential for the order parameters in a (partially) bosonized formulation $[17,68,69]$. Thus, if the size of all four-quark couplings is found to be bounded in the RG flow, the system remains in the symmetric regime [17,35-37,67] and our ansatz (18) for the effective action provides us with an approximate description of QCD on all scales. The observation of a divergent four-quark coupling for a given temperature and quark chemical potential may hence be considered an indicator that the order-parameter potential develops a nontrivial ground-state expectation value in the direction associated with a specific four-quark channel. In this case, our ansatz (18) for the effective action now provides us only with an approximate description of QCD for scales above the one at which a divergent fourquark coupling is observed. The critical temperature $T_{\mathrm{cr}}(\mu)$ at a given value of the quark chemical potential above which no spontaneous symmetry breaking occurs is then defined as the smallest temperature for which the fourquark couplings still remain finite in the infrared (IR) limit associated with $k \rightarrow 0$ [15-17,36,37]. However, we emphasize that our present approach is only able to detect phase transitions of second order as the definition of the critical temperature is associated with a change from positive to negative curvature of the order-parameter potential at the origin. In the case of a first-order phase transition, a nontrivial minimum of the potential is formed, but the curvature at the origin remains positive. Consequently, our criterion for the detection of a phase transition does not allow us to detect first-order transitions. Still, it allows us to detect the line of metastability [15]; for the first NJL model analysis of this aspect, see [70]. In any case, the nontrivial assumption entering our analysis of the QCD phase structure in the present work is that it is possible to relate the dominance pattern of the four-quark couplings to the symmetry-breaking pattern in terms of condensates; see Refs. $[15,16,67,71]$ for a detailed discussion of this aspect.

It is reasonable to expect that the symmetry-breaking patterns associated with the various four-quark channels change when we vary, e.g., the quark chemical potential. For example, the diquark channel may become more relevant than the scalar-pseudoscalar channel at high density. The most dominant channel can be identified by requiring that the modulus of the coupling of this channel is greater than the ones of all the other four-quark couplings. For such an analysis to be meaningful, it is therefore ultimately required to consider a Fierz-complete set of four-quark couplings.

For the derivation of the RG flow equations of the fourquark couplings, we employ the Wetterich equation [51], which is a RG equation for the quantum effective action $\Gamma$. Within this framework, the effective action $\Gamma$ depends on the RG scale $k$, which is related to the so-called RG "time" $t=\ln (k / \Lambda)$. Note that the scale $k$ defines an infrared (IR) cutoff scale, and $\Lambda$ may be chosen to be the scale at which we fix the initial conditions of the RG flow of the 


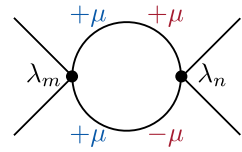

(a)

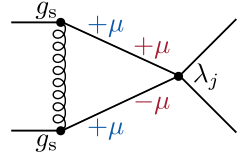

(b)

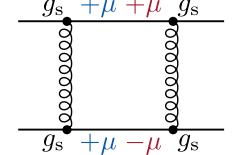

(c)
FIG. 1. The 1PI diagrams contributing to the RG flow (19) of the four-quark couplings $\lambda_{i}$. Note that there are two classes of each diagram, which are associated with the signs of the quark chemical potential appearing in the quark propagators: One class represents the case of equal signs as depicted by the blue labels, and the other one represents the case of opposite signs as depicted by the red labels.

couplings. The general structure of the RG flow equations for the dimensionless renormalized couplings $\lambda_{i}=Z_{i} k^{2} \bar{\lambda}_{i}$ is given by

$\partial_{t} \lambda_{i}=2 \lambda_{i}-\mathcal{A}_{m n}^{(i)}\left(\tau, \tilde{\mu}_{\tau}\right) \lambda_{m} \lambda_{n}-\mathcal{B}_{j}^{(i)}\left(\tau, \tilde{\mu}_{\tau}\right) \lambda_{j} g_{\mathrm{s}}^{2}-\mathcal{C}^{(i)}\left(\tau, \tilde{\mu}_{\tau}\right) g_{\mathrm{s}}^{4}$,

with $\tau=T / k$ and $\tilde{\mu}_{\tau}=\mu /(2 \pi T)$. The temperature- and chemical-potential-dependent coefficients $\mathcal{A}_{m n}^{(i)}, \mathcal{B}_{j}^{(i)}$, and $\mathcal{C}^{(i)}$ are auxiliary functions containing sums of the 1PI diagrams depicted in Fig. 1. In addition, these functions also contain the information on the chosen gauge. For convenience, we restrict ourselves to Feynman gauge in the following. In Eq. (19), terms bilinear in the four-quark couplings with the coefficients $\mathcal{A}_{m n}^{(i)}$ are associated with the purely fermionic diagrams (a) in Fig. 1. Terms proportional to $\lambda_{j} g_{\mathrm{s}}^{2}$ are generated by the triangle diagrams (b) depicted in Fig. 1. Finally, terms proportional to $g_{\mathrm{s}}^{4}$ are associated with the box diagrams (c) shown in Fig. 1. Note that we have dropped an explicit dependence of these loop diagrams on the anomalous dimensions of the quark and gluon fields as these additional contributions have been found to be subleading in the symmetric regime [32,35-37,72].

For the derivation of the set of RG flow equations (19), we have made extensive use of existing software packages $[73,74]$. Because of the size of the resulting system of equations for the Fierz-complete set of four-quark couplings, we deal with the flow equations only numerically and therefore refrain from listing these equations explicitly here. An explicit representation of the flow equations for the purely fermionic part as parametrized by the matrices $\mathcal{A}_{m n}^{(i)}$ can be found in our preceding work [16], including a discussion of the regularization scheme also underlying our present work. For the same regularization scheme, an explicit representation of a Fierz-complete set of flow equations for the four-quark interactions in the vacuum limit in case of an $S U\left(N_{\mathrm{c}}\right) \otimes U_{\mathrm{L}}(2) \otimes U_{\mathrm{R}}(2)$ symmetry can be found in Ref. [35], where the contributions proportional to $\lambda_{j} g_{\mathrm{s}}^{2}$ and $g_{\mathrm{s}}^{4}$ have been taken into account as well.
In our present approximation, the RG flow of the gauge sector enters the flow equations of the four-quark couplings only via the running of the strong coupling. In our numerical analysis in Sec. III, we employ the running coupling computed nonperturbatively in Refs. [36,37] where the same regularization scheme has been used as in the present work. Let us be more explicit at this point. In Refs. [36,37], the running of the gauge coupling has been computed at zero and finite temperature but not at finite quark chemical potential. An extension of these nonperturbative studies to finite quark chemical potential is beyond the scope of the present work and therefore deferred to future work. To estimate the effect of a quark chemical potential on the running of the strong coupling and thereby on the flow of the four-quark couplings, we employ the running strong coupling from Refs. [36,37] as obtained for (pure) Yang-Mills $(\mathrm{YM})$ theory $\left(N_{\mathrm{f}}=0\right)$ and for QCD with two massless quark flavors. The reason behind this is that, at the one-loop level, the quark contribution to the $\beta$ function of the strong coupling at finite quark chemical potential and zero temperature has been found to be identical to the one in the vacuum limit for RG scales $k \geq \mu$ and to vanish identically for $k<\mu$; see, e.g., Refs. $[41,50,75]$. This implies that, at least at the one-loop level at zero temperature, the RG flow of the strong coupling is identical to the one in the vacuum limit for $k \geq \mu$ but identical to the one in YM theory for $k<\mu$. Loosely speaking, the YM coupling and the QCD coupling in the vacuum limit may therefore be viewed as two extremes of the zero-temperature QCD coupling at finite quark chemical potential. The stepwise change of the $\beta$ function of the strong coupling at $k=\mu$ is then smeared out at finite temperature.

In addition to corrections to the strong coupling originating from the presence of a finite quark chemical potential, one may be worried about possible corrections to the strong coupling arising from the presence of quark self-interactions. However, provided the flow of the four-quark couplings is governed by the presence of fixed points [72], as is the case in the symmetric regime [35-37], it follows from the analysis of (modified) WardTakahashi identities that the backreaction of the four-quark couplings on the strong coupling is negligible. For our present analysis, the use of the strong coupling from Refs. [36,37] in our set of flow equations for the fourquark couplings is therefore not only a convenient but also a consistent approximation.

We close this discussion by adding that the mechanism underlying the dynamical generation of the four-quark couplings and the role of the running gauge coupling for spontaneous symmetry breaking can already be understood in simple terms by analytically analyzing the fixed-point structure of the RG flow equations for the four-quark couplings. Here, we refrain from repeating this general line of arguments and only refer the reader to the discussions given in Refs. [35-37,76-78]; see Ref. [17] for an introduction. 


\section{Scale-fixing procedure}

As already mentioned above, the initial values of the four-quark interaction channels are not considered as fundamental parameters in our present approach and are set to zero at the UV scale $\Lambda$. This corresponds to a $U_{\mathrm{A}}(1)$ symmetric scenario according to the sum rules (16) and (17). The case with explicit $U_{\mathrm{A}}(1)$-symmetry breaking is discussed separately in Sec. III B. In any case, the fourquark couplings must be generated dynamically by quarkgluon interactions, which removes a potential bias in the parameter choice as present in low-energy model studies. In fact, within our present study, we predict the values of the four-quark couplings from the underlying quark-gluon dynamics. This is a feature that has already been used in Ref. [14] to constrain the equation of state of symmetric nuclear matter.

The only free parameter in our study is the value of the running gauge coupling $g_{\mathrm{s}}$ at the initial RG scale $\Lambda$. This value is adjusted at the UV scale $\Lambda=10 \mathrm{GeV}$ to obtain a critical temperature $T_{\mathrm{cr}}(\mu=0) \equiv T_{0}=132 \mathrm{MeV}$ at zero quark chemical potential, which is the value of the chiral phase transition temperature at $\mu=0$ found in very recent lattice QCD studies [79]. ${ }^{3}$ In Sec. III, we use the scale $T_{0}$ as a reference scale; i.e., we "measure" all physical observables in units of this scale. The chosen value for $\Lambda$ ensures that $T / \Lambda \ll 1$ and $\mu / \Lambda \ll 1$ for the range of external parameters considered in the present work and therefore allows us to avoid cutoff artifacts (and to reduce regularization-scheme dependence) [27].

In order to obtain the critical temperature $T_{\mathrm{cr}}(\mu=0)=$ $132 \mathrm{MeV}$ at $\mu=0$, we tune the running gauge coupling at the initial scale $\Lambda$. For example, for our study with a gauge coupling with two quark flavors, this amounts to choosing $\alpha_{\mathrm{s}}(\Lambda=10 \mathrm{GeV})=g_{\mathrm{s}}^{2} /(4 \pi)=0.2137$. Evolved to the $Z$-boson mass scale $M_{Z}=91.19 \mathrm{GeV}$, the value of the gauge coupling fixed in this way is then about $6 \%$ greater than the experimental results [80]. The necessity of a larger initial value for the running gauge coupling in order to trigger criticality in the quark sector has been observed before in studies employing approximations of the present type; see, e.g., Ref. [45]. Only the most advanced RG truncations with a very accurate treatment of momentum structures do not require such an "enhancement"; see, e.g., Refs. [20,21]. The latter is of particular importance for a quantitative description of low-energy observables within the symmetry-broken regime. For our present work aiming at a study of the onset of spontaneous symmetry breaking and an analysis of symmetry breaking patterns, we expect that this plays a secondary role.

\footnotetext{
${ }^{3}$ The lattice QCD study presented in Ref. [79] considering two degenerate massless quarks and a physical strange quark mass finds the chiral phase transition $T_{\mathrm{cr}}=132_{-6}^{+3} \mathrm{MeV}$ at zero quark chemical potential.
}

Finally, we note that we have checked that our results are robust with respect to a variation of the initial RG scale $\Lambda$. To be specific, our results are found to converge when we choose $\Lambda \gtrsim 2 \mathrm{GeV}$, provided that the gauge coupling at this initial scale has been tuned correspondingly. Note that this not only holds for the position of the computed phase boundaries but also for the dominance patterns of the fourquark couplings [for both our $U_{\mathrm{A}}(1)$-symmetric studies and for those with explicit $U_{\mathrm{A}}(1)$ breaking].

\section{PHASE DIAGRAM}

\section{A. Symmetry-breaking patterns}

Let us now study the phase diagram in the plane spanned by the temperature and the quark chemical potential for the $U_{\mathrm{A}}(1)$-symmetric case, i.e., with all four-quark couplings set to zero at the initial RG scale $\Lambda$. Recall that we have defined the critical temperature $T_{\mathrm{cr}}(\mu)$ at a given value of the quark chemical potential to be the smallest temperature for which all four-quark couplings still remain finite in the IR limit $k \rightarrow 0$ [15-17,36,37]. Also keep in mind that a divergence in the flow of one four-quark coupling at a critical scale $k_{\mathrm{cr}}(T, \mu)$ entails corresponding divergences in all other channels as well. However, as discussed in detail in Refs. [15,16], the four-quark couplings, in general, develop distinct relative strengths, and it is possible to identify a dominant fourquark channel; i.e., the modulus of the associated coupling is (significantly) greater than the absolute values of all the other four-quark couplings (see our general discussion above).

As a first nontrivial result, we observe a dominance of the scalar-pseudoscalar coupling in the vacuum limit $(T=\mu=0)$, with the couplings diverging at a symmetrybreaking scale $k_{\mathrm{cr}}(\mu=0) / T_{0} \approx 2.62$. Close to the symmetry-breaking scale, we find that the modulus of the scalar-pseudoscalar coupling is at least 2 times greater than the modulus of all other couplings, suggesting that the QCD ground state is governed by chiral symmetry breaking in the vacuum limit. At first glance, this is very similar to the situation observed in NJL- and quark-mesontype studies. However, the crucial difference is given by the initial conditions for the four-quark couplings. In the present case, we can exclude that the dominance of the scalar-pseudoscalar channel may have been triggered by the choice of the initial conditions of the four-quark couplings since they are set to zero at the initial scale and are therefore only generated dynamically. Thus, the dominance of the scalar-pseudoscalar coupling indicating chiral symmetry breaking is solely generated by quarkgluon dynamics. This is in line with earlier RG flow studies of QCD in the vacuum limit [19,20], and it has indeed been observed that this dominance entails the formation of a corresponding chiral condensate [20,21] governing the low-energy dynamics. 


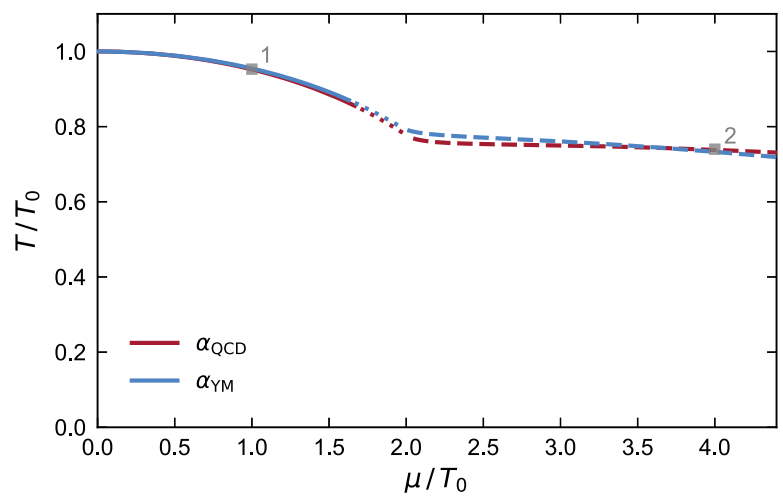

FIG. 2. Phase boundary associated with the spontaneous breakdown of at least one of the fundamental symmetries of QCD as accessible by our Fierz-complete ansatz (red and blue lines). The red line has been obtained by employing a strong coupling with two massless quark flavors. To illustrate the effect of quark contributions to the strong coupling, we also show the phase boundary as obtained when the strong coupling from pure YangMills theory is employed; see main text for details. The gray boxes labeled " 1 " and "2" specify two exemplary points for which the RG flows of the four-quark couplings are shown in Fig. 3.

Increasing the temperature at vanishing quark chemical potential, the dominance of the scalar-pseudoscalar interaction channel persists even up to high temperatures beyond the critical temperature. The red line in Fig. 2 depicts the critical temperature as a function of the quark chemical potential which has been obtained with the running gauge coupling as computed in Refs. [36,37] for
$N_{\mathrm{f}}=2$, here denoted by $\alpha_{\mathrm{QCD}}$. Following the phase boundary from small to large chemical potential, we first observe that the dominance of the scalar-pseudoscalar interaction channel persists up to $\mu / T_{0} \approx 1.7$ as indicated by the solid red line. To illustrate the relative strengths of the various four-quark couplings in this regime, we show the scale dependence of the (dimensionful) renormalized couplings at $\mu / T_{0}=1.0$ for $T / T_{0} \approx 0.95 \gtrsim T_{\text {cr }}(\mu) / T_{0}$ (i.e., right above the phase transition) in the left panel of Fig. 3. This point is indicated in the phase diagram by the little gray box labeled "1." For purposes of illustration, the various couplings are normalized by the value of the scalar-pseudoscalar coupling $\bar{\lambda}_{(\sigma-\pi)}$ at $k=0$. We readily observe that the dynamics is clearly dominated by the scalar-pseudoscalar coupling. In fact, its modulus is at least 2 times greater than the modulus of all other couplings. According to our line of argument, this dominance indicates that in this regime the phase boundary continues to be governed by chiral symmetry breaking.

Following the phase transition line, we then encounter that the exclusive dominance of the scalar-pseudoscalar channel goes away, and a small region from approximately $\mu / T_{0} \approx 1.7$ to $\mu / T_{0}=\mu_{\chi} / T_{0} \approx 2.0$ opens up (as depicted by the red dotted line in Fig. 2). In this region, we observe that the scalar-pseudoscalar channel, the CSC channel, as well as the $(S+P)_{-}^{\text {adj }_{-},},(V+A)_{\|}^{\text {adj }}{ }_{-}$, and $(V-A)_{\perp}^{\text {adj }}$ channels are most dominant, meaning that these channels are significantly greater compared to the remaining interaction channels. Such a region of "mixed" dominance might potentially indicate a metastable or mixed phase [71].

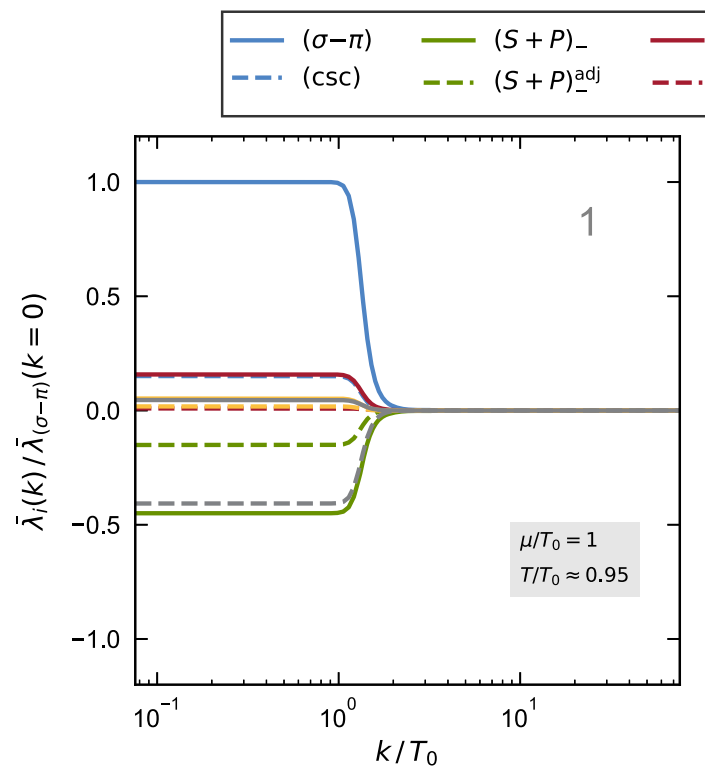

$\begin{array}{lllll}(V+A)_{\|} & =(V-A)_{\|} & - & (V+A)_{\|}^{\mathrm{adj}} \\ (V+A)_{\perp} & -- & (V-A)_{\perp} & --\cdot(V-A)_{\perp}^{\mathrm{adj}}\end{array}$

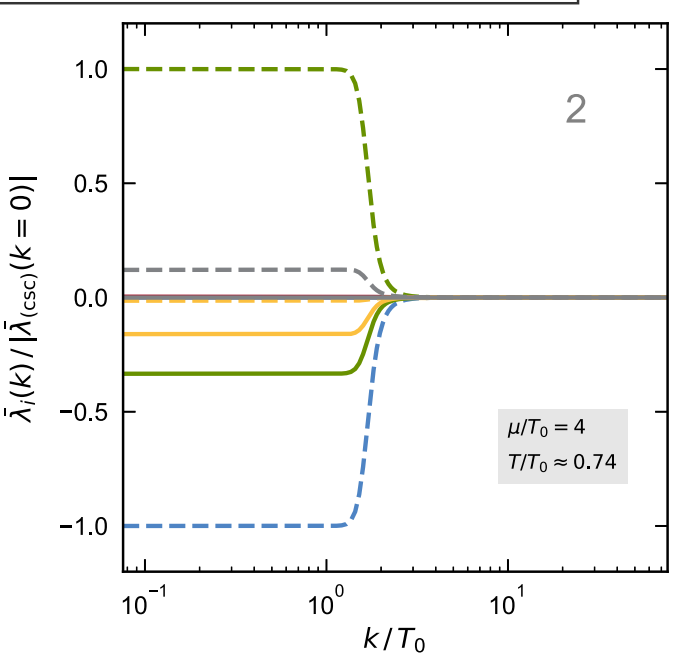

FIG. 3. Scale dependence of the various renormalized (dimensionful) four-quark couplings at $\mu / T_{0}=1$ and $T / T_{0} \approx 0.95$ (left panel), as well as at $\mu / T_{0}=4.0$ and $T / T_{0} \approx 0.74$ (right panel). These two parameter sets correspond to the little gray boxes in the phase diagram shown in Fig. 2. According to the sum rules (16) and (17), the intact $U_{\mathrm{A}}(1)$ symmetry implies $\bar{\lambda}_{\mathrm{csc}}=-\bar{\lambda}_{(S+P)_{-}^{\mathrm{adj}}}$, in agreement with our numerical results. 
It is noteworthy that four out of this set of five dominant channels are adjoint interaction channels, which may point to a nontrivial color structure of the ground state in this regime. However, the appearance of this regime may also very well be a consequence of the $U_{\mathrm{A}}(1)$-preserving initial conditions as the resulting $U_{\mathrm{A}}(1)$-symmetric $\mathrm{RG}$ flow affects the development of dominance. Indeed, the "entanglement" of several equally strong four-quark couplings is lifted by taking into account $U_{\mathrm{A}}(1)$-violating effects; see Sec. III B.

For $\mu / T_{0} \gtrsim 2.0$, depicted by the red dashed line in Fig. 2, we eventually observe a clear dominance of the CSC channel, indicating the emergence of a diquark condensate $\delta^{a}$. This dominance is again illustrated by the scale dependence of the couplings in this region shown in the right panel of Fig. 3. There, we show the RG flow for $\mu / T_{0}=4.0$ and $T / T_{0} \approx 0.74 \gtrsim T_{\text {cr }}(\mu) / T_{0}$ (i.e., right above the phase transition). In the phase diagram, the corresponding point is depicted by the little gray box labeled " 2 " in Fig. 3. Note that, in the right panel of Fig. 3, the couplings are now normalized by the modulus of the dominant CSC coupling, $\left|\bar{\lambda}_{\text {csc }}\right|$ at $k=0$. We observe that the modulus of any other four-quark coupling is at most less than half the value of the CSC coupling, except for the $(S+P)_{-}^{\text {adj }}$ coupling. The latter assumes the same value in the IR as the CSC coupling, only with opposite sign. The reason for this behavior is that the boundary conditions with all fourquark couplings initially set to zero at the UV scale $\Lambda$ leave the axial $U_{\mathrm{A}}(1)$ symmetry intact, as already mentioned above. Since the RG flow preserves the symmetries of the initial effective average action, the sum rules (16) and (17) are exactly fulfilled at all scales $k$, with the first sum rule

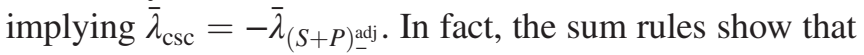
two of the ten four-quark couplings of our Fierz-complete basis are not independent in the $U_{\mathrm{A}}(1)$-symmetric case. We discuss the effect of $U_{\mathrm{A}}(1)$-violating initial discussions in Sec. III B.

In Fig. 4, we show the dominance pattern of the fourquark couplings along the finite-temperature phase boundary presented in Fig. 2 (as obtained with the two-flavor coupling $\alpha_{\mathrm{QCD}}$ ). The values of the dimensionful renormalized couplings are shown for $k \rightarrow 0$ as functions of the quark chemical potential for temperatures right above the critical temperature $T_{\mathrm{cr}}(\mu)$, where we have normalized the couplings by the scalar-pseudoscalar coupling $\bar{\lambda}_{(\sigma-\pi)}$ for $\mu=0$ at $k=0$ for convenience. As already mentioned above, we first observe a clear dominance of the scalarpseudoscalar interaction channel for $\mu / T_{0} \lesssim 1.7$, followed by a change of the dominance pattern to a dominance of the CSC coupling for $\mu / T_{0} \gtrsim 2$. In the region of CSC dominance, the intact $U_{\mathrm{A}}(1)$ symmetry is again encoded by the fact that the values of the CSC and the $(S+P)_{-}^{\text {adj }}$ coupling are identical, up to their signs. The dominance of the CSC coupling for $\mu / T_{0} \gtrsim 2$ is as clearly visible as the dominance of the scalar-pseudoscalar coupling for $\mu / T_{0} \lesssim 1$.7. In fact,

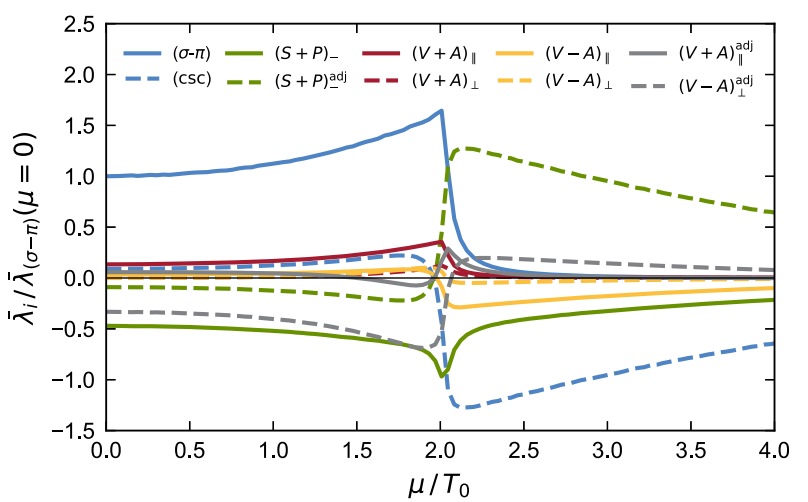

FIG. 4. (Dimensionful) renormalized four-quark couplings at $k=0$ as functions of the quark chemical potential for temperatures $\left(T-T_{\mathrm{cr}}(\mu)\right) / T_{0} \approx 0.004$ (i.e., slightly above the respective critical temperature $T_{\mathrm{cr}}$ ), illustrating the "hierarchy" of the four-quark couplings in terms of their relative strength along the phase boundary (i.e., along the red line in Fig. 2). For convenience, the values of the four-quark couplings are normalized by the value of the scalar-pseudoscalar coupling $\bar{\lambda}_{(\sigma-\pi)}$ for $\mu=0$ at $k=0$.

the ratio of the modulus of the second largest coupling and the largest coupling is even smaller in this highdensity regime. Note that, in Fig. 4, the values of the four-quark couplings are extracted at a temperature $T$ slightly above the critical temperature $T_{\mathrm{cr}}(\mu)$, where $\left(T-T_{\text {cr }}(\mu)\right) / T_{0} \approx 0.004$. By this, we ensure that the RG flow is fully located in the symmetric regime, and the flow can be followed down to $k=0$. However, owing to this small distance to the phase transition line, the aforementioned region with "mixed" dominance is not fully resolved in this figure.

The distinct dominance and evident "hierarchy" among the four-quark self-interactions in the low- and high-density regime are nicely illustrated in Fig. 4. In view of this result, it is tempting to speculate whether such a change in the hierarchy points to the existence of a nearby tricritical point in the phase diagram. Yet, this aspect is speculative as the presently employed approximations do not allow a definite answer to this question. However, we emphasize that the change in the hierarchy from a dominance of the scalarpseudoscalar coupling to a dominance of the CSC coupling at $\mu_{\chi} / T_{0} \approx 2.0$ is a nontrivial outcome of our study as it is completely determined by the dynamics of the system itself. The four-quark couplings are initially set to zero at the UV scale $\Lambda$ and are dynamically generated by quarkgluon dynamics in the RG flow. Thus, the dynamics is not affected by any kind of fine-tuning of the boundary conditions of the four-quark couplings which would potentially favor particular channels.

At this point, let us once more note that the dominance of a four-quark coupling only indicates the onset of the formation of an associated condensate. It neither guarantees the actual formation (e.g., IR fluctuations could restore the 
associated symmetries) nor does it strictly exclude the possible formation of other condensates associated with subdominant couplings. Our analysis based on the dominance pattern of the four-quark couplings must therefore be taken with some care; see also our discussion in our preceding works $[15,16]$. Still, in the context of condensedmatter physics, the appearance of a clear dominance of a given channel has been found to be a precursor of the formation of a corresponding condensate [71].

In Fig. 2, we also included results for the finitetemperature phase boundary from a computation where we have used a running gauge coupling as obtained in YM theory (depicted by the blue line labeled $\alpha_{\mathrm{YM}}$ ). The phase boundary as well as the dominance agree almost perfectly with the results from the computation using the running coupling $\alpha_{\mathrm{QCD}}$. To be specific, we observe a dominance of the scalar-pseudoscalar coupling at small quark chemical potential, a regime characterized by a "mixed" dominance pattern between $1.7 \lesssim \mu / T_{0} \lesssim 2.0$, and finally a clear dominance of the CSC coupling at large quark chemical potential. This is noteworthy since the YM coupling $\alpha_{\mathrm{YM}}$ grows more rapidly than the QCD coupling $\alpha_{\mathrm{QCD}}$ when the scale $k$ is lowered. However, the effect of this difference in the scale dependence is also, to a certain extent, compensated by our scale-fixing procedure. The initial value of the YM gauge coupling has to be smaller than for the $\alpha_{\mathrm{QCD}}$ coupling in order to obtain the same critical temperature $T_{0} \equiv T_{\text {cr }}(\mu=0)=132 \mathrm{MeV}$ at zero quark chemical potential. Nevertheless, this observation may be viewed as an indication that the hierarchy of the various couplings in terms of their relative strength is predominantly determined by the dynamics within the quark sector, whereas the gauge sector as associated with the details of the running coupling is mostly required to bring the quark sector close to criticality in the first place. Once the gauge sector has rendered the four-quark couplings sufficiently large, they become relevant operators in the RG flow, and the quarks start to develop their "own effective dynamics." Then, the details of the gauge sector play a subleading role, at least in the present approximation. Loosely speaking, one may therefore state that we are basically left with a NJL-type model once the four-quark couplings have been rendered sufficiently large by the underlying quark-gluon dynamics.

\section{B. Role of $U_{\mathrm{A}}(1)$ symmetry}

The initial conditions of the RG flow chosen so far leave the axial $U_{\mathrm{A}}(1)$ symmetry intact. All couplings of the fourquark self-interactions are set to zero at the UV scale $\Lambda$ and are solely generated dynamically by quark-gluon interactions in the RG flow. In, e.g., Refs. [36,37], $U_{\mathrm{A}}(1)-$ violating channels have been omitted based on the assumption that they become relevant only in the lowenergy regime governed by spontaneous symmetry breaking. Our Fierz-complete basis $\mathcal{B}$ composed of the ten fourquark interaction channels is effectively reduced to eight interaction channels in the case of the $U_{\mathrm{A}}(1)$ symmetry being intact [16]. Recall that the sum rules (16) and (17) imply that two of the couplings associated with the four $U_{\mathrm{A}}(1)$-violating interaction channels of our basis $\mathcal{B}$ are not independent. These sum rules are exactly fulfilled at all scales in the symmetric phase and for all $k>k_{\mathrm{cr}}$ in the phase governed by spontaneous symmetry breaking. In the latter case, the $U_{\mathrm{A}}(1)$ symmetry may potentially still be broken spontaneously below the symmetry-breaking scale $k_{\mathrm{cr}}$. However, this cannot be resolved within our present approximation.

In our preceding study of the phase structure of the NJL model and the role of Fierz completeness [16], we have observed that $U_{\mathrm{A}}(1)$ symmetry breaking affects the dominance of the four-quark couplings in terms of their relative strength along the finite-temperature phase boundary. In particular, we have found explicit $U_{\mathrm{A}}(1)$ symmetry breaking to be important for the formation of the conventional CSC ground state at intermediate and large values of the chemical potential. In this work, the four-quark couplings are now dynamically generated by quark-gluon dynamics. Following the critical temperature $T_{\mathrm{cr}}(\mu)$ as a function of the quark chemical potential, we observe two regions characterized by different distinct hierarchies of the fourquark couplings which are remarkably robust against a variation of the running gauge coupling; see Fig. 2 for the $U_{\mathrm{A}}(1)$-symmetric case. The scalar-pseudoscalar interaction channel dominates the dynamics at small quark chemical potential, signaling the formation of the chiral condensate, whereas at higher quark chemical potential, the dominance of the conventional CSC coupling suggests the formation of a diquark condensate. The latter is observed in spite of the intact $U_{\mathrm{A}}(1)$ symmetry in our considerations thus far. Only for $1.7 \lesssim \mu / T_{0} \lesssim 2.0$, we observe a regime which is characterized by several equally strong four-quark interaction channels; see our discussion in the previous subsection. Moreover, the dominance of the CSC coupling is always accompanied by an equally strong $(S+P)_{-}^{\text {adj }}$ channel as a direct consequence of the intact $U_{\mathrm{A}}(1)$ symmetry: The sum rule (16) ties the modulus of the CSC coupling to the modulus of the $(S+P)_{-}^{\text {adj }}$ coupling.

In order to probe the role of explicit $U_{\mathrm{A}}(1)$-symmetry breaking in our present study, we now analyze the RG flow for $U_{\mathrm{A}}(1)$-violating boundary conditions for the four-quark couplings. The strength of $U_{\mathrm{A}}(1)$ breaking is effectively controlled by the initial value of the $(S+P)_{\text {_ }}$ coupling since the associated four-quark interaction channel is directly related to the so-called 't Hooft determinant (14); see Refs. [3,58-63]. In the following, we therefore vary only the initial condition of this coupling but still set the initial values of the other four-quark couplings to zero at the UV scale $\Lambda$. For a given initial value of the $(S+P)_{-}$ coupling, we then adjust the UV value of the gauge coupling $g_{\mathrm{s}}(\Lambda)$ such that the value of the critical temperature at zero chemical potential remains unchanged, 

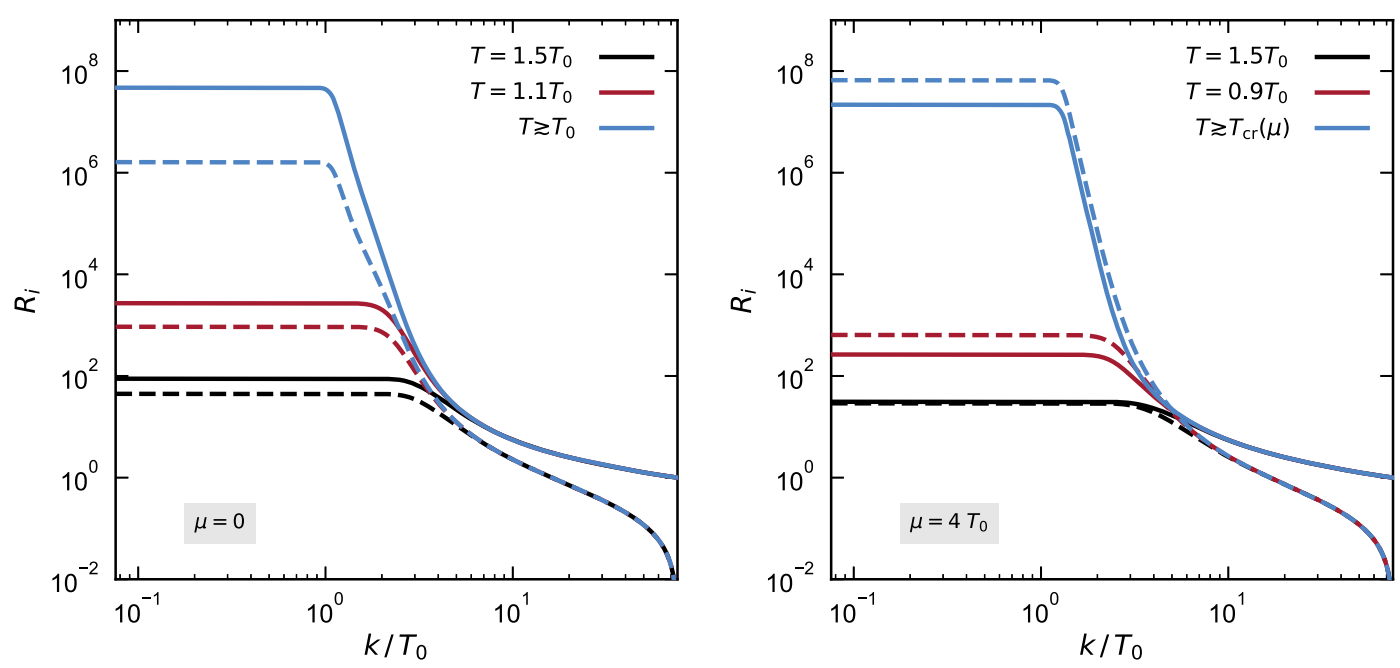

FIG. 5. Illustration of the scale dependence of the explicit $U_{\mathrm{A}}(1)$ breaking as measured by the normalized sum rules $R_{1}$ (dashed lines) and $R_{2}$ (solid lines) at $\mu=0$ (left panel) and at $\mu / T_{0}=4.0$ (right panel) for three values of the temperature for each of the two cases. The strength of the initial explicit $U_{\mathrm{A}}(1)$ symmetry breaking is controlled by the value of the (dimensionless) renormalized coupling of the $(S+P)_{-}$channel ('t Hooft channel) at the UV scale $\Lambda$, exemplarily chosen to be $\lambda_{(S+P)_{-}}^{(\mathrm{UV})}=1.0$ in this figure.

$T_{\text {cr }}(\mu=0)=132 \mathrm{MeV}$. This ensures comparability between our results for different initial conditions for the $(S+P)_{-}$coupling.

As also done in Refs. [15,16], we begin by analyzing the fate of the $U_{\mathrm{A}}(1)$ symmetry at finite temperature and quark chemical potential with the aid of the sum rules (16) and (17). To this end, we first normalize the two sum rules as follows:

$$
1=\left.\left(R_{1}+R_{2}\right)\right|_{k=\Lambda}
$$

where

$$
R_{i}=\mathcal{N}\left|\mathcal{S}_{U_{\mathrm{A}}(1)}^{(i)}\right|
$$

The quantities $\mathcal{S}_{U_{\mathrm{A}}(1)}^{(1)}$ and $\mathcal{S}_{U_{\mathrm{A}}(1)}^{(2)}$ are defined in Eqs. (16) and (17), respectively. A strong deviation of the sum rules from zero indicates strong effective $U_{\mathrm{A}}(1)$ breaking. In Fig. 5, we show the scale dependence of the sum rules at two characteristic values of the chemical potential for three values of the temperature for each of the two cases. Interestingly, we observe the exact same qualitative behavior as found in Ref. [16] for the NJL model. At small quark chemical potential close to the critical temperature $T_{\mathrm{cr}}(\mu)$, $U_{\mathrm{A}}(1)$ breaking is driven by the scalar-pseudoscalar channel associated with pion dynamics, and it becomes stronger toward the IR as indicated by increasing values of $R_{1}$ and $R_{2}$, with $R_{2} \gg R_{1}$. At large chemical potential, we find that the strength of $U_{\mathrm{A}}(1)$-symmetry breaking also becomes stronger as the phase boundary is approached from above, but it is now driven by the dynamics of diquark d.o.f. as associated with the CSC channel. As a consequence, $R_{1}$ and $R_{2}$ are of the same order of magnitude since both depend on the CSC coupling; see Eqs. (16) and (17). In either case, for increasing temperature, $U_{\mathrm{A}}(1)$ breaking as measured by the sum rules remains more and more on its initial level as determined by the $U_{\mathrm{A}}(1)$ violating boundary conditions in the UV regime since quark fluctuations become more and more thermally suppressed.

Let us now compare the phase diagram as obtained with the $U_{\mathrm{A}}(1)$-symmetric initial conditions employed in the previous subsection, i.e., with all four-quark couplings initially set to zero, to the phase diagrams resulting from $U_{\mathrm{A}}(1)$-violating initial conditions. The strength of the explicit $U_{\mathrm{A}}(1)$ breaking at the initial UV scale $\Lambda$ is controlled by the value of the (dimensionless) renormalized coupling of the $(S+P)_{-}$channel ('t Hooft channel), which we choose to assume the values $\lambda_{(S+P)_{-}}^{(\mathrm{UV})}=0.01,0.1,1.0$. In the following, we only present results from computations using the running gauge coupling for two massless quark flavors. As in the $U_{\mathrm{A}}(1)$-symmetric case, the dependence on our specific choice for the coupling is found to be very mild anyhow. In Fig. 6, the various phase diagrams as obtained with these three different choices for the boundary conditions are shown. It is remarkable how little the critical phase temperature as a function of the quark chemical potential is affected by the strength of the initial explicit breaking of the $U_{\mathrm{A}}(1)$ symmetry, although the strength in terms of the initial value of the $(S+P)_{-}$coupling is varied over 3 orders of magnitude. ${ }^{4}$ Across the entire range of chemical potentials shown in this figure, the variation of the

\footnotetext{
${ }^{4}$ Note that the considered range of values for the $(S+P)_{-}$ coupling is consistent with the size of the value that is expected from a direct computation of this quantity at a given scale $\Lambda$ [63].
} 


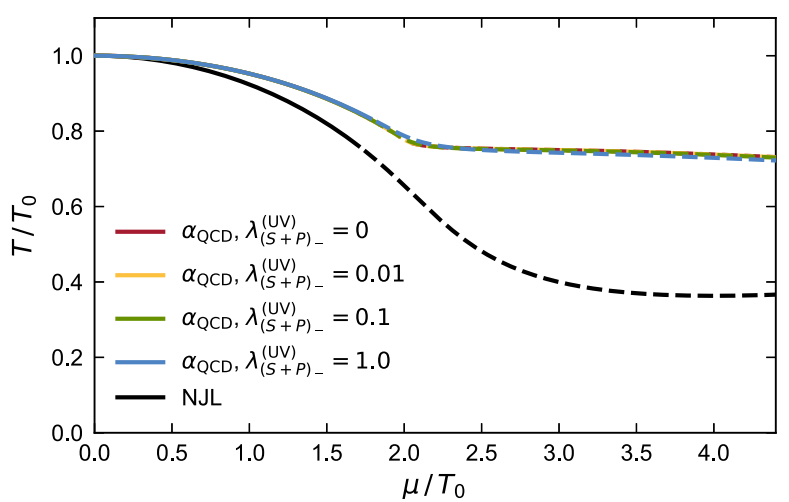

FIG. 6. Phase boundary associated with the spontaneous breakdown of at least one of the fundamental symmetries of QCD as accessible by our Fierz-complete ansatz now under the influence of explicit $U_{\mathrm{A}}(1)$ symmetry breaking, in comparison to the phase boundary resulting from a corresponding Fierz-complete NJL model study (black line); see also Ref. [16]. All results (except for those from the NJL model study) have been obtained by employing a strong coupling with two massless quark flavors. The strength of the initial explicit $U_{\mathrm{A}}(1)$ symmetry breaking is controlled by the value of the (dimensionless) renormalized coupling of the $(S+P)_{\text {_ }}$ channel ('t Hooft channel) at the UV scale $\Lambda$. The values of all other four-quark couplings have been chosen to be initially zero. The phase boundary is shown for $U_{\mathrm{A}}(1)$-symmetric boundary conditions as well as for $U_{\mathrm{A}}(1)$ violating initial conditions with $\lambda_{(S+P)_{-}}^{(\mathrm{UV})_{1}}=0.01,0.1,1.0$. A dominance of the scalar-pseudoscalar interaction channel is depicted by solid lines and a dominance of the CSC channel by dashed lines. The case of "mixed" dominance occurring for $U_{\mathrm{A}}(1)$-symmetric boundary conditions is indicated by a dotted line, although it is hardly visible on the scale of the plot.

critical temperature for any given value of the chemical potential is less than $2 \%{ }^{5}$ In all cases, we observe a dominance of the scalar-pseudoscalar coupling at small quark chemical potential, depicted by the solid lines in Fig. 6. The regime of "mixed" dominance for chemical potentials in the range $1.7 \lesssim \mu / T_{0} \lesssim 2.0$ appearing in the case of $U_{\mathrm{A}}(1)$-symmetric initial conditions vanishes for all considered $U_{\mathrm{A}}(1)$-violating initial conditions. Choosing $0.01 \leq \lambda_{(S+P)_{-}}^{(\mathrm{UV})_{\mathrm{A}}} \leq 1.0$ for the initial value of the coupling associated with the $U_{\mathrm{A}}(1)$-violating $(S+P)_{-}$channel, we indeed find that the dominance changes directly from the scalar-pseudoscalar channel to the CSC channel within the region $1.8 \lesssim \mu_{\chi} / T_{0} \lesssim 2.0$, as indicated by the dashed lines in Fig. 6. This is similar to what has been found in a Fierzcomplete NJL model study [16].

\footnotetext{
${ }^{5}$ For all initial conditions of the computations shown in Fig. 6, the symmetry-breaking scale in the vacuum limit remains approximately the same value, $k_{\mathrm{cr}} / T_{0} \approx 2.6$. Still, a direct quantitative comparison of the phase boundaries has to be taken with some care as the different computations do not necessarily lead to the same values of low-energy observables.
}

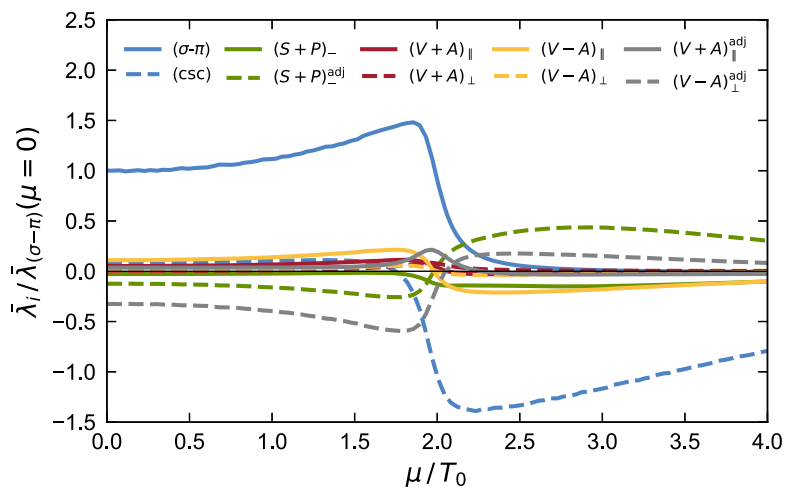

FIG. 7. Hierarchy of the four-quark couplings in terms of their relative strength along the phase boundary (blue line in Fig. 6). To obtain this figure, we have evaluated the renormalized fourquark couplings for $k \rightarrow 0$ as functions of the quark chemical potential for temperatures $\left(T-T_{\mathrm{cr}}(\mu)\right) / T_{0} \approx 0.002$ [i.e., slightly above the critical temperature $T_{\mathrm{cr}}(\mu)$ for a given quark chemical potential]. The values of the four-quark couplings are shown for $U_{\mathrm{A}}(1)$-violating initial conditions with $\lambda_{(S+P)_{-}}^{(\mathrm{UV})_{1}}=1.0$ for the 't Hooft coupling. To normalize the couplings, we use the scalarpseudoscalar coupling for $k \rightarrow 0$ and zero quark chemical potential.

The hierarchy of the various four-quark couplings in terms of their relative strength along the phase boundary is shown in Fig. 7, exemplarily for the initial coupling $\lambda_{(S+P)_{-}}^{(\mathrm{UV})_{-}}=1.0$. This figure again shows the values of the (dimensionful) renormalized couplings for $k \rightarrow 0$ as functions of the quark chemical potential for temperatures $\left(T-T_{\mathrm{cr}}(\mu)\right) / T_{0} \approx 0.002$ [i.e., slightly above the critical temperature $T_{\mathrm{cr}}(\mu)$ for a given quark chemical potential $\mu$ ]. The values are normalized by the scalar-pseudoscalar coupling $\bar{\lambda}_{(\sigma-\pi)}$ for $k \rightarrow 0$ at zero chemical potential. We again observe a clear dominance of the scalar-pseudoscalar interaction channel for $\mu / T_{0} \lesssim 2.0$ and a clear dominance of the CSC interaction channel for larger values of the chemical potential. Compared to the case with $U_{\mathrm{A}}(1)$ symmetric boundary conditions (see Fig. 4), these dominances appear even more pronounced in the present case. In particular, the CSC channel is not accompanied anymore by an equally strong $(S+P)_{-}^{\text {adj }}$ coupling. The latter now assumes considerably smaller values for $\mu / T_{0} \gtrsim 2.0$ in comparison to the computation with intact $U_{\mathrm{A}}(1)$ symmetry, whereas the CSC coupling assumes even slightly larger values. From this, we conclude that the breaking of the $U_{A}(1)$ symmetry plays an important role in "shaping the hierarchy" of the four-quark interaction channels and thus in the formation of associated condensates as indicated by their dominances. This observation confirms the importance of explicit $U_{\mathrm{A}}(1)$ breaking for the formation of the conventional CSC ground state at large chemical potential as already discussed in Ref. [16] for NJL-type models. In this respect, we also refer again to early seminal works on color superconductivity; see, e.g., Refs. [81-90]. It is 
noteworthy that we find that the change in the hierarchy from a dominance of the scalar-pseudoscalar coupling to a dominance of the CSC coupling at $\mu_{\chi} / T_{0} \approx 2.0$ is remarkably insensitive to the initial strength of explicit $U_{\mathrm{A}}(1)$ symmetry breaking as controlled by the initial coupling $\lambda_{(S+P)_{-}}^{(\mathrm{UV})}$ associated with the 't Hooft channel. Whereas the aforementioned insensitivity of the position of the phase boundary may be partially attributed to the scale-fixing procedure underlying our study, we find that the observed change in the hierarchy of four-quark couplings and the sole dominance of the CSC coupling triggered only by the explicit $U_{\mathrm{A}}(1)$ symmetry breaking is a nontrivial outcome completely determined by the dynamics of the system itself.

Let us finally compare our results with those from a Fierz-complete NJL model study [16]. In Fig. 6, we also show the finite-temperature phase boundary resulting from a Fierz-complete NJL model study (black line). The corresponding flow equations have been presented in Ref. [16]. In this computation, the initial scalarpseudoscalar coupling at the UV scale $\Lambda / T_{0} \approx 75.76$ has been tuned such that we obtain $T_{\mathrm{cr}}(\mu=0)=132 \mathrm{MeV}$ for the critical temperature at $\mu=0$. The remaining initial fourquark couplings are set to zero. Interestingly, we find that the finite-temperature phase boundary agrees well with the one obtained from our present study with a two-flavor running gauge coupling $\alpha_{\mathrm{QCD}}$, at least for small quark chemical potential. For $\mu / T_{0} \gtrsim 0.5$, however, the two phase boundaries start to deviate from each other significantly. Indeed, at the largest quark chemical potential shown in Fig. 6, $\mu / T_{0}=4.4$, the critical temperature resulting from the NJL model computation is $T_{\mathrm{cr}} / T_{0} \approx 0.366$. In contrast to that, at the same quark chemical potential, the computation including dynamical gauge fields yields a critical temperature that exceeds the one from the NJL model study by a factor of $2: T_{\mathrm{cr}}\left(\mu=4.4 T_{0}\right) / T_{0} \approx 0.731$. This observation may have further phenomenological consequences. For example, in standard BCS theory, the critical temperature can be directly related to the size of the diquark gap $\delta^{a}$ at zero temperature, i.e., $T_{\mathrm{cr}} \sim|\delta|[26,57,91]$. Thus, the observed increase of the critical temperature in our results from the computation including dynamical gauge d.o.f. may hint at a larger diquark gap at $T=0$.

Recall that we use different initial conditions for the four-quark couplings in our NJL model study and in our study with dynamical gauge fields. This is required since different mechanisms are at play which drive the quark sector to criticality. To be specific, in our NJL model study, it is required to choose a sufficiently large initial scalarpseudoscalar coupling to ensure that the RG flow diverges at a finite symmetry-breaking scale $k_{\mathrm{cr}}$ for sufficiently low temperatures, signaling the onset of spontaneous symmetry breaking. Apart from the value of the coupling associated with the 't Hooft channel, the four-quark couplings in our QCD study are solely dynamically generated, and the quark sector is driven to criticality by the gauge coupling becoming sufficiently large; see Refs. [17,35-37] for a detailed discussion of the latter mechanism. Of course, this difference also affects the results at large quark chemical potential, although the gauge coupling plays a less prominent role in this regime as the dynamics is largely controlled by the appearance of a BCS-type instability. One may argue that the initial conditions chosen in the case of the NJL model study actually favor the scalarpseudoscalar coupling and do not sufficiently support the dynamics associated with the formation of a diquark condensate or other channels which may become relevant at large chemical potential. Indeed, the boundary conditions enforce that the dynamics are initially driven by the scalar-pseudoscalar self-interaction, at least over a wide range of RG scales. Still, at large chemical potential, the CSC channel is found to dominate the dynamics even in the case of the NJL model. As discussed in detail in Refs. [16], this can be understood from an analysis of the fixed-point structure. Our study taking into account gauge d.o.f. comes without the requirement of an initial tuning of a specific four-quark coupling. It is therefore intriguing that the "hierarchy" of the various interaction channels in both computations changes at approximately the same quark chemical potential from the scalar-pseudoscalar coupling to the CSC coupling; see Fig. 6. This observation indicates that the hierarchy of the interaction channels in terms of their strength is determined, to a large extent, by the interplay of the various four-quark couplings themselves; see also our discussion in the previous subsection.

As a closing remark, we would like to add that the comparison of the different phase boundaries shown in Figs. 2 and 6 have to be taken with some care. Although all computations yield approximately the same critical scale $k_{\mathrm{cr}} / T_{0} \approx 2.6$ (symmetry-breaking scale) in the vacuum limit, which sets the scale for low-energy observables $\mathcal{O} \sim$ $k_{\text {cr }}$ (see, e.g., Ref. [76]), our present approximation does not allow us to check whether the different studies indeed lead to the same values of low-energy observables in the IR limit. This potential issue complicates a comparison of our results for the phase boundary, as well as our subsequent comparison of the curvature of the phase boundary.

\section{Curvature of the phase boundary}

Finally, we briefly comment on the curvature $\kappa$ of the finitetemperature phase boundary at small chemical potential:

$$
\kappa=-\left.T_{0} \frac{\mathrm{d} T_{\mathrm{cr}}(\mu)}{\mathrm{d} \mu^{2}}\right|_{\mu=0} .
$$

The results for the curvature as obtained from various studies are summarized in Table I.

Compared to our Fierz-complete NJL model study, we find the curvature to be significantly decreased in our study, including dynamical gauge d.o.f. In fact, the curvature is 
TABLE I. Curvature $\kappa$ of the two-flavor finite-temperature phase boundary as obtained from different studies; see main text for details. Note that the curvature range for the mean-field studies reflects the difference between the chiral limit and the case of physical pion masses. The fRG results in this work have been obtained in the chiral limit.

\begin{tabular}{lc}
\hline \hline Setting & Curvature $\kappa$ \\
\hline Mean field (NJL, one channel) $[9,92]$ & $0.197 \ldots 0.200$ \\
fRG (NJL, Fierz-complete) & 0.074 \\
fRG (QCD, $U_{\mathrm{A}}(1)$-symmetric) & 0.046 \\
fRG (QCD) & 0.046 \\
Lattice QCD [93-96] & $0.034 \ldots 0.070$ \\
\hline \hline
\end{tabular}

reduced by approximately $40 \%$. The curvature of a standard one-channel NJL model study in the mean-field approximation is even more than 4 times greater than that in our present study with gauge d.o.f. This holds true for all settings with gauge d.o.f. considered in this work, including computations using a Yang-Mills coupling, a strong coupling for the two-flavor case, as well as computations taking into account explicit $U_{\mathrm{A}}(1)$ symmetry breaking [labeled "fRG (QCD)" in Table I]. Note that the results from our Fierz-complete fRG calculations including gauge d.o.f. listed in Table I have been obtained with the strong coupling for the two-flavor case. For the case with explicit $U_{\mathrm{A}}(1)$ symmetry breaking, we have chosen $\lambda_{(S+P)_{-}}^{(\mathrm{UV})}=1.0$ for the initial condition of the coupling associated with the 't Hooft channel. A summary of results for the curvature from lattice QCD calculations with two flavors can also be found in Table I. We observe that the results from our Fierzcomplete studies taking into account gauge d.o.f. are well in accordance with those from lattice QCD studies. We add that low-energy model studies indicate that the inclusion of IR fluctuation effects tend to further lower the value of the curvature [92,97-99]. For the 2+1-flavor case, more recent lattice results for the curvature are also available, indicating $\kappa \sim 0.034 \ldots 0.21$ [100]. The curvature found in a very recent $2+1$-flavor fRG-QCD study with physical masses is also in accordance with the latter results [50]. In any case, a direct comparison of our present results to those from $2+1$-flavor studies is only possible to a limited extent, if at all. Still, in general, it is reasonable to expect, from our present study, that the issue of Fierz incompleteness also affects the results for the curvature in the $2+1$-flavor case, as it does in the 2-flavor case (see Table I). Indeed, it has also been observed in a Fierz-incomplete two-channel study of a $2+1$-flavor NJL-type model that four-quark interaction channels other than the scalar-pseudoscalar channel can significantly impact the value of the curvature [101].

\section{CONCLUSIONS}

In this work, we have analyzed the RG flow of fourquark interactions in the pointlike limit in a Fierz-complete fashion starting from the classical QCD action in the UV limit. Working in the chiral limit, the only parameter of our study in the $U_{\mathrm{A}}(1)$-symmetric limit is given by the strong coupling $g_{\mathrm{s}}$ which we fixed at a large initial UV scale in the perturbative regime.

With this setting at hand, we found that the inclusion of gluodynamics leads to an increase of the critical temperatures at large quark chemical potential in comparison to the results from a corresponding Fierz-complete NJL model study. Assuming that the critical temperature can be related to the size of the zero-temperature gap in a color superconducting phase of quark matter, our results therefore suggest that the diquark gap is likely to be greater than the one found in corresponding NJL model studies, at least within the range of chemical potentials considered in our present work.

Toward the IR limit, the treatment of the four-quark interactions in the pointlike limit does not allow us to access the phase governed by spontaneous symmetry breaking. The introduction of mesonic auxiliary fields by means of a Hubbard-Stratonovich transformation or by applying the more advanced technique of dynamical hadronization (see Refs. [32,52,69,102] and, e.g., Refs. [20,21,39,45,50] for their application to QCD) would enable us to study the dynamics even within regimes governed by spontaneous symmetry breaking. The use of the latter technique would allow us to continuously perform Hubbard-Stratonovich transformations of the fourquark couplings in the RG flow (at every RG scale) and would thereby enable us to access the low-energy regime, i.e., the regime below the symmetry-breaking scale $k_{\mathrm{cr}}$ in our present study, including a computation of the orderparameter potential. In particular, with respect to a more detailed study of the effect of $U_{\mathrm{A}}(1)$ breaking in the low-energy regime, it may be worthwhile to build this technique around a combination of our present study and existing detailed functional RG studies of the lowenergy sector taking $U_{\mathrm{A}}(1)$ breaking into account $[103,104]$. Nevertheless, in order to gain some insight into the structure of the ground state emerging in the case of spontaneous symmetry breaking already within our present study, we followed the approach of our NJL model studies in Refs. [15,16] and analyzed the hierarchy of the fourquark couplings.

Our RG analysis of the $U_{\mathrm{A}}(1)$-symmetric case revealed a clear dominance of the scalar-pseudoscalar interaction channel associated with chiral symmetry breaking at small quark chemical potential. Very importantly, this dominance is not triggered by a specific choice for the initial conditions of the four-quark couplings since all four-quark couplings are set to zero at the initial RG scale; i.e., they are solely gluon induced in the RG flow. For $\mu / T_{0} \gtrsim 2.0$, we then observe a change in the hierarchy. In this regime, the CSC channel associated with the formation of the most conventional color superconducting condensate in two-flavor 
QCD now dominates the quark dynamics. We emphasize that the dominance pattern as a function of the quark chemical potential as well as the actual position $\mu_{\chi}$, where the dominance pattern changes from a scalar-pseudoscalar dominance to a CSC dominance, is found to be a remarkably robust feature. The little influence of the different running gauge couplings considered may be viewed as an indication that the dominance pattern is largely determined within the quark sector. The gauge sector as associated with the details of the running coupling is mostly required to bring the quarks close to criticality. Once the four-quark couplings have been rendered sufficiently large by the underlying quark-gluon dynamics, the quarks develop their "own dynamics," and the details of the gauge sector start to play a subleading role, at least in the present approximation. At this point, loosely speaking, we are then basically left with a NJL-type model. Note again that the dynamics in our present study is not "contaminated" by any kind of fine-tuning of the initial conditions for the four-quark couplings, which would, in general, favor particular channels. However, the analysis based on the hierarchy of the four-quark couplings must nevertheless be taken with some care. In fact, it is clear that a dominance of a specific four-quark coupling neither guarantees the formation of an associated condensate in the IR nor does it exclude the formation of other condensates. Still, it should also be mentioned that corresponding studies in the context of condensed-matter theory show that the appearance of a clear dominance of a specific four-fermion coupling is indeed a precursor for the formation of the associated condensate [71].

In order to probe the effect of explicit $U_{\mathrm{A}}(1)$-symmetry breaking on the phase boundary and the dominance pattern of the four-quark couplings, we implemented $U_{\mathrm{A}}(1)$ violating initial conditions in the form of finite values for the coupling associated with the so-called 't Hooft channel. Even at large chemical potential, the considered strengths of explicit $U_{\mathrm{A}}(1)$ symmetry breaking at the initial UV scale showed surprisingly little effect on the shape of the phase boundary. The same is true for the actual position of the point $\mu_{\chi}$, where the dominance pattern of the fourquark couplings changes qualitatively. Compared to the $U_{\mathrm{A}}(1)$-symmetric $\mathrm{RG}$ flow, however, $U_{\mathrm{A}}(1)$-violating initial conditions affect the hierarchy of the four-quark couplings along the phase boundary, which is clearly visible in, e.g., the amplification of the dominance of the scalar-pseudoscalar interaction channel at small chemical potential as well as of the dominance of the CSC channel at large chemical potential. From this, we conclude that explicit $U_{\mathrm{A}}(1)$ symmetry breaking indeed plays an important role in the formation of the condensates, in particular, with respect to the formation of the conventional CSC ground state at large chemical potential [81-90]. Future extensions of our present work should of course include a direct computation of the 't Hooft coupling within our RG flow by following earlier works in this direction [63].

Of course, our present study can be further improved in various directions. Still, our analysis already provides an important insight into the dynamics underlying the QCD phase structure at finite temperature and quark chemical potential and consolidates the findings obtained from our preceding Fierz-complete NJL-type model study [16]. Moreover, the inclusion of gauge d.o.f. combined with the Fierz-complete set of four-quark interactions enables us to identify the relevant effective low-energy d.o.f. and to determine, or at least constrain, from first principles the couplings of a suitably constructed truncation for the low-energy sector, in particular, at large quark chemical potential. In the future, this may prove very valuable, e.g., to study the thermodynamics of quark matter at high density. Indeed, first steps in this direction have already been taken very recently [14].

\section{ACKNOWLEDGMENTS}

The authors would like to thank Markus Q. Huber, J. M. Pawlowski, and D. Rosenblüh for useful discussions and J. M. Pawlowski also for comments on the manuscript. This work has been done within the fQCD Collaboration [105]. J. B. acknowledges support by the DFG under Grant No. BR 4005/4-1 (Heisenberg program). J. B. acknowledges support by the Helmholtz International Center for the Facility for Antiproton and Ion Research (HIC for FAIR) within the LOEWE program of the State of Hesse. This work is supported by the Deutsche Forschungsgemeinschaft (DFG, German Research Foundation)—Projektnummer 279384907-SFB 1245.
[1] Y. Nambu and G. Jona-Lasinio, Phys. Rev. 122, 345 (1961).

[2] Y. Nambu and G. Jona-Lasinio, Phys. Rev. 124, 246 (1961).

[3] S. P. Klevansky, Rev. Mod. Phys. 64, 649 (1992).
[4] T. Hatsuda and T. Kunihiro, Phys. Rep. 247, 221 (1994).

[5] J. Berges, N. Tetradis, and C. Wetterich, Phys. Rep. 363, 223 (2002).

[6] B.-J. Schaefer and J. Wambach, Phys. Part. Nucl. 39, 1025 (2008). 
[7] L. von Smekal, Nucl. Phys. B, Proc. Suppl. 228, 179 (2012).

[8] M. Drews and W. Weise, Prog. Part. Nucl. Phys. 93, 69 (2017).

[9] M. Buballa, Phys. Rep. 407, 205 (2005).

[10] K. Fukushima, J. Phys. G 39, 013101 (2012).

[11] M. G. Alford, A. Schmitt, K. Rajagopal, and T. Schäfer, Rev. Mod. Phys. 80, 1455 (2008).

[12] R. Anglani, R. Casalbuoni, M. Ciminale, N. Ippolito, R. Gatto, M. Mannarelli, and M. Ruggieri, Rev. Mod. Phys. 86, 509 (2014).

[13] A. L. Watts, N. Andersson, D. Chakrabarty, M. Feroci, K. Hebeler, G. Israel, F. K. Lamb, M. C. Miller, S. Morsink, F. Özel, A. Patruno, J. Poutanen, D. Psaltis, A. Schwenk, A. W. Steiner, L. Stella, L. Tolos, and M. van der Klis, Rev. Mod. Phys. 88, 021001 (2016).

[14] M. Leonhardt, M. Pospiech, B. Schallmo, J. Braun, C. Drischler, K. Hebeler, and A. Schwenk, arXiv:1907. 05814.

[15] J. Braun, M. Leonhardt, and M. Pospiech, Phys. Rev. D 96, 076003 (2017).

[16] J. Braun, M. Leonhardt, and M. Pospiech, Phys. Rev. D 97, 076010 (2018).

[17] J. Braun, J. Phys. G 39, 033001 (2012).

[18] J. Braun and T. K. Herbst, arXiv:1205.0779.

[19] J. Braun, Functional renormalization group methods in quantum chromodynamics, Ph. D. thesis, Heidelberg University, 2006.

[20] M. Mitter, J. M. Pawlowski, and N. Strodthoff, Phys. Rev. D 91, 054035 (2015).

[21] A. K. Cyrol, M. Mitter, J. M. Pawlowski, andN. Strodthoff, Phys. Rev. D 97, 054006 (2018).

[22] T. Hatsuda and T. Kunihiro, Phys. Rev. Lett. 55, 158 (1985).

[23] B.-J. Schaefer and M. Wagner, Phys. Rev. D 79, 014018 (2009).

[24] Z. Zhang and T. Kunihiro, Phys. Rev. D 80, 014015 (2009).

[25] P. Springer, J. Braun, S. Rechenberger, andF. Rennecke, EPJ Web Conf. 137, 03022 (2017).

[26] D. Bailin and A. Love, Phys. Rep. 107, 325 (1984).

[27] J. Braun, M. Leonhardt, and J. M. Pawlowski, SciPost Phys. 6, 056 (2019).

[28] J. Braun, K. Schwenzer, and H.-J. Pirner, Phys. Rev. D 70, 085016 (2004).

[29] T. K. Herbst, M. Mitter, J. M. Pawlowski, B.-J. Schaefer, and R. Stiele, Phys. Lett. B 731, 248 (2014).

[30] E. Meggiolaro and C. Wetterich, Nucl. Phys. B606, 337 (2001).

[31] H. Gies, Phys. Rev. D 66, 025006 (2002).

[32] H. Gies and C. Wetterich, Phys. Rev. D 69, 025001 (2004).

[33] J. M. Pawlowski, D. F. Litim, S. Nedelko, and L. von Smekal, Phys. Rev. Lett. 93, 152002 (2004).

[34] C. S. Fischer and H. Gies, J. High Energy Phys. 10 (2004) 048.

[35] H. Gies and J. Jaeckel, Eur. Phys. J. C 46, 433 (2006).

[36] J. Braun and H. Gies, Phys. Lett. B 645, 53 (2007).

[37] J. Braun and H. Gies, J. High Energy Phys. 06 (2006) 024.

[38] J. Braun, H. Gies, and J. M. Pawlowski, Phys. Lett. B 684, 262 (2010).
[39] J. Braun, Eur. Phys. J. C 64, 459 (2009).

[40] F. Marhauser and J. M. Pawlowski, arXiv:0812.1144.

[41] J. Braun, L. M. Haas, F. Marhauser, and J. M. Pawlowski, Phys. Rev. Lett. 106, 022002 (2011).

[42] J. Braun, A. Eichhorn, H. Gies, and J. M. Pawlowski, Eur. Phys. J. C 70, 689 (2010).

[43] K.-I. Kondo, Phys. Rev. D 82, 065024 (2010).

[44] L. Fister and J. M. Pawlowski, Phys. Rev. D 88, 045010 (2013).

[45] J. Braun, L. Fister, J. M. Pawlowski, and F. Rennecke, Phys. Rev. D 94, 034016 (2016).

[46] F. Rennecke, Phys. Rev. D 92, 076012 (2015).

[47] A. K. Cyrol, L. Fister, M. Mitter, J. M. Pawlowski, and N. Strodthoff, Phys. Rev. D 94, 054005 (2016).

[48] A. K. Cyrol, M. Mitter, J. M. Pawlowski, and N. Strodthoff, Phys. Rev. D 97, 054015 (2018).

[49] L. Corell, A. K. Cyrol, M. Mitter, J. M. Pawlowski, and N. Strodthoff, SciPost Phys. 5, 066 (2018).

[50] W.-J. Fu, J. M. Pawlowski, and F. Rennecke, arXiv:1909. 02991.

[51] C. Wetterich, Phys. Lett. B 301, 90 (1993).

[52] J. M. Pawlowski, Ann. Phys. (Amsterdam) 322, 2831 (2007).

[53] H. Gies, Lect. Notes Phys. 852, 287 (2012).

[54] N. Khan, J. M. Pawlowski, F. Rennecke, and M. M. Scherer, arXiv:1512.03673.

[55] W.-j. Fu, J. M. Pawlowski, F. Rennecke, and B.-J. Schaefer, Phys. Rev. D 94, 116020 (2016).

[56] K. Fukushima and T. Hatsuda, Rep. Prog. Phys. 74, 014001 (2011).

[57] A. Altland and B. Simons, Condensed Matter Field Theory (Cambridge University Press, Cambridge, England, 2006).

[58] G. 't Hooft, Phys. Rev. D 14, 3432 (1976); Erratum, 18, 2199 (1978)

[59] G. 't Hooft, Phys. Rev. Lett. 37, 8 (1976).

[60] M. A. Shifman, A. I. Vainshtein, and V. I. Zakharov, Nucl. Phys. B163, 46 (1980).

[61] E. V. Shuryak, Nucl. Phys. B203, 93 (1982).

[62] T. Schäfer and E. V. Shuryak, Rev. Mod. Phys. 70, 323 (1998).

[63] J. M. Pawlowski, Phys. Rev. D 58, 045011 (1998).

[64] T. Kunihiro, AIP Conf. Proc. 1235, 23 (2010).

[65] M. Kobayashi and T. Maskawa, Prog. Theor. Phys. 44, 1422 (1970).

[66] M. Kobayashi, H. Kondo, and T. Maskawa, Prog. Theor. Phys. 45, 1955 (1971).

[67] J. Braun, H. Gies, L. Janssen, and D. Roscher, Phys. Rev. D 90, 036002 (2014).

[68] U. Ellwanger and C. Wetterich, Nucl. Phys. B423, 137 (1994).

[69] H. Gies and C. Wetterich, Phys. Rev. D 65, 065001 (2002).

[70] M. Asakawa and K. Yazaki, Nucl. Phys. A504, 668 (1989).

[71] D. Roscher, N. Gneist, M. M. Scherer, S. Trebst, and S. Diehl, Phys. Rev. B 100, 125130 (2019).

[72] H. Gies, J. Jaeckel, and C. Wetterich, Phys. Rev. D 69, 105008 (2004).

[73] M. Q. Huber and J. Braun, Comput. Phys. Commun. 183, 1290 (2012).

[74] A. K. Cyrol, M. Mitter, and N. Strodthoff, Comput. Phys. Commun. 219, 346 (2017). 
[75] P. Springer, Constraining low-energy models of QCD from fundamental interactions, Ph. D. thesis, TU Munich, 2016.

[76] J. Braun and H. Gies, J. High Energy Phys. 05 (2010) 060.

[77] J. Braun, C. S. Fischer, and H. Gies, Phys. Rev. D 84, 034045 (2011).

[78] Y. Kusafuka and H. Terao, Phys. Rev. D 84, 125006 (2011).

[79] H. T. Ding et al., Phys. Rev. Lett. 123, 062002 (2019).

[80] M. Tanabashi et al. (Particle Data Group), Phys. Rev. D 98 , 030001 (2018).

[81] M. G. Alford, K. Rajagopal, and F. Wilczek, Phys. Lett. B 422, 247 (1998).

[82] R. Rapp, T. Schäfer, E. V. Shuryak, and M. Velkovsky, Phys. Rev. Lett. 81, 53 (1998).

[83] J. Berges and K. Rajagopal, Nucl. Phys. B538, 215 (1999).

[84] D. T. Son, Phys. Rev. D 59, 094019 (1999).

[85] R. D. Pisarski and D. H. Rischke, Phys. Rev. D 61, 074017 (2000).

[86] R. D. Pisarski and D. H. Rischke, Phys. Rev. D 61, 051501 (2000).

[87] T. Schäfer and F. Wilczek, Phys. Rev. D 60, 114033 (1999).

[88] W. E. Brown, J. T. Liu, and H.-C. Ren, Phys. Rev. D 61, 114012 (2000).

[89] D. K. Hong, V. A. Miransky, I. A. Shovkovy, and L. C. R. Wijewardhana, Phys. Rev. D 61, 056001 (2000); Erratum, 62, 059903 (2000).

[90] N. J. Evans, J. Hormuzdiar, S. D. H. Hsu, and M. Schwetz, Nucl. Phys. B581, 391 (2000).

[91] A. Schmitt, Q. Wang, and D. H. Rischke, Phys. Rev. D 66, 114010 (2002).
[92] K.-I. Aoki, S.-I. Kumamoto, and M. Yamada, Nucl. Phys. B931, 105 (2018).

[93] P. de Forcrand and O. Philipsen, Nucl. Phys. B642, 290 (2002).

[94] C. R. Allton, S. Ejiri, S. J. Hands, O. Kaczmarek, F. Karsch, E. Laermann, and C. Schmidt, Phys. Rev. D 68, 014507 (2003).

[95] L.-K. Wu, X.-Q. Luo, and H.-S. Chen, Phys. Rev. D 76, 034505 (2007).

[96] O. Philipsen, Eur. Phys. J. ST 152, 29 (2007).

[97] B.-J. Schaefer and J. Wambach, Nucl. Phys. A757, 479 (2005).

[98] J. Braun, B. Klein, and B.-J. Schaefer, Phys. Lett. B 713, 216 (2012).

[99] J. M. Pawlowski and F. Rennecke, Phys. Rev. D 90, 076002 (2014).

[100] M. D’Elia, Nucl. Phys. A982, 99 (2019).

[101] N. M. Bratovic, T. Hatsuda, and W. Weise, Phys. Lett. B 719, 131 (2013).

[102] S. Floerchinger and C. Wetterich, Phys. Lett. B 680, 371 (2009).

[103] D. U. Jungnickel and C. Wetterich, Phys. Rev. D 53, 5142 (1996).

[104] J. Berges, D. U. Jungnickel, and C. Wetterich, Phys. Rev. D 59, 034010 (1999).

[105] J. Braun, L. Corell, A. K. Cyrol, W.-J. Fu, M. Leonhardt, M. Mitter, J. M. Pawlowski, M. Pospiech, F. Rennecke, N. Wink (fQCD Collaboration) (members as of January 2018). 